\title{
Similarity Solutions of Jet Development Mixing Layers Using Algebraic and 1-Equation Turbulence Models
}

\begin{abstract}
V. Tesař
Mixing layers are formed between two parallel fluid streams having different velocities. One of the velocities may be zero, as is the usual case of the mixing layer that surrounds, immediately downstream from the nozzle, the core of a developing jet issuing into stagnant surroundings. Earlier - but so far not properly published - experimental evidence shows a remarkably weak effect of transversal curvature, making the present solution applicable with acceptable precision to description of developing round jets. This paper presents solutions of a planar mixing layer by a similarity transformation, which reduces the problem to solving ordinary differential equations. Two solutions are investigated: one based on an algebraic model and the other using the 1-equation model of turbulence. They are compared with recent results of PIV measurements of a developing jet.
\end{abstract}

Keywords: similarity of shear flows, mixing layer, turbulence models, algebraic model, one-equation model, submerged jets.

\section{Introduction}

\subsection{Similarity solutions of shear flows}

Fluid flows are governed by the Navier-Stokes partial differential equation, for which there are (apart from a handful of cases of trivial simplicity) no practically useful analytical solutions. The equation is therefore usually solved by numerical procedures over a domain discretised into finite elements or volumes. The numerical solutions do not provide a global view of the problem, because - in contrast to the general character of analytical solutions - each computed case is valid only for a particular set of parameters and boundary condition values, not showing a relation with other cases. This is acceptable for solving a particular engineering task, but not helpful for educational purposes or for general investigations.

Sometimes it is possible to obtain solutions having the desirable general character by utilising a similarity property [9] of the flowfield. There are not many flows possessing this property. Fortunately, many basic cases of shear flows do: the spatial distributions of their flow parameters - e.g., the velocity profiles - at different streamwise locations are mutually similar so that the introduction of suitably transformed co-ordinates can make them identical. The resultant universal velocity profile in transformed co-ordinates then represents all profiles at all streamwise locations in the flowfield. The similarity transformation reduces the number of independent variables in the problem. The governing partial differential equations are reduced to ordinary differential equations in the transformed co-ordinates. This transformation approach was first used by G. Stokes who obtained analytical solutions for unsteady boundary layers developing in time-dependent motion of flat walls [2]. Later this use of the symmetry properties of governing equations became the standard tool for studies of laminar shear flows by L. Prandtl and his Göttingen school. To this day, standard teaching of laminar boundary layer theory is based on this idea, using the solution [2] obtained under Prandtl's guidance by Blasius in 1908. Another researcher influenced by Prandtl, Schlichting in 1933, used this approach for laminar submerged jets [2] and Glauert in
1956 applied it successfully to the laminar wall jet problem [2].

Modern approach to the similarity transformations are based on the ideas of E. Noether [16] who proved that each conservation law of a physical problem is associated with symmetry of the governing equations. The treatment of the relationship between physical invariants and Lie-Bäcklund operators, which are Noether symmetries as shown by Kara and Mahomed [17], was influenced by the group theory ideas of Ibragimov [18].

Application to turbulent shear flows has been slowed by problems of modelling turbulence. In 1926, W. Tollmien [1] applied the similarity approach to submerged turbulent jets using Prandtl's 1925 algebraic model of turbulence [2]. This model requires independently input information about the size of the turbulent vortices. Tollmien supplied this in the form of a very simple assumption: the turbulence length scale was assumed to be constant at each cross section and proportional to the local width of the shear flow. His results were, unfortunately, in only very rough agreement with experimental data. The reason for the disagreement, the non-local character of turbulence transported by advection as well as diffusion, was initially not recognised and the remedy was sought in introduction of several other physically not substantiated algebraic turbulence models. In particular, current textbooks still often discuss turbulent submerged jets on the basis of Görtler's 1942 [8] solution based on Prandtl's conceptually wrong 1942 "neues Modell". The popularity of this solution is due - besides its simplicity - to purely fortuitous better agreement with experimental data than obtained with Tollimen's solution. Only at the end of the last century did similarity solutions of fully developed turbulent jets using advanced models, taking into account turbulence transport, become available - an example are the solutions of turbulent jets by Tesař 1996 [3], 1995 [5], 1997 [9], 2001 [6]. Essential problem to be overcome is the complexity of modern turbulence models. To incorporate the transport effects the solved equation for fluid momentum needs simultaneous solution of additional transport equations for parameters of turbulence. The less complex one-equation turbulence model used in [3] 
relies on the original hypothesis about the size of the momentum transporting turbulent vortices as originally introduced by Tollmien [1]. The more sophisticated two-equation model used in [5] does not require any such a priori assumption. The solutions in [3] and [5] assume the turbulence to be isotropic, which is not satisfied exactly but fortunately the anisotropy in the jet flows is not very large. Agreement with experiments is excellent for both models, which shows the Tollmien's simple length scale hypothesis to be remarkably successful.

Both solutions [3] and [5] assume the jet to be fully developed. Experiments show this causes more problems than generally believed. According to many standard textbooks, jets are said to be fully developed at a downstream distance as short as 8 to 10 nozzle exit widths (or diameters in the axisymmetric case). This is based on the character of velocity profiles, which at these locations indeed agree with the similarity predictions reasonably well. However, parameters of turbulence require a much longer distance to develop. Profiles of fluctuation energy were found by Tesař and Stř́lka in [6] to be not fully developed at a downstream distance as large as 60 diameters - so large that the jet may cease there to be useful for practical applications (e.g. due to the velocity of an air jet decreasing to a level comparable with room draft motions).

\subsection{Mixing layer}

Elimination of the streamwise distance variable in shear flows by the similarity transformation is achieved by dividing the transverse distances by the shear layer thickness $d$ and dividing the velocity by the difference between the highest and lowest velocity. In practical situations it is extremely rare for the transversal dimensions of solid walls defining the flow geometry to vary in the streamwise direction in the same way as the layer thickness. This means that practical condition for the existence of similarity solutions is the absence of scale defining solid walls.

The mixing layer, Fig. 1, between two parallel flows having different velocities $w_{e A}$ and $w_{e B}$, away from the bounding walls, is an example of such a scale-less geometry. Of particular importance are the cases with $w_{e B}=0$, i.e. the mixing layer between a single stream and stagnant surroundings. An important fact in Fig. 1 is the planar character of the geometry.

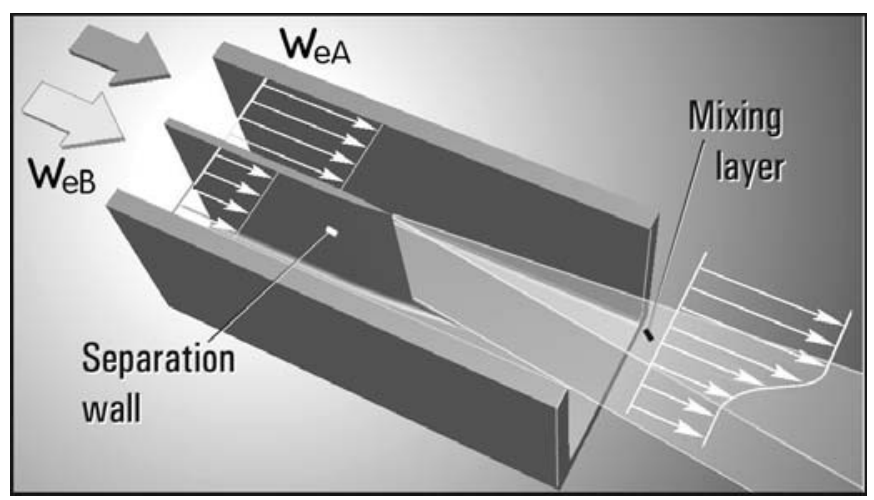

Fig. 1: Mixing layer between parallel flows is quite thin because momentum diffusion from the flow of higher velocity $w_{e A}$ towards the lower velocity $w_{e B}$ is slower than the streamwise motion. The character of the flow is here represented by time-mean velocity profile.
The related axisymmetric case shown in Fig. 2 is not scale-less. There is the transversal dimension - the radius of transversal curvature $r_{0}$ of the edge of the separating wall. Since the transversal curvature does not vary equally as the layer thickness with the streamwise distances, this flow ceases to possess exact similarity.

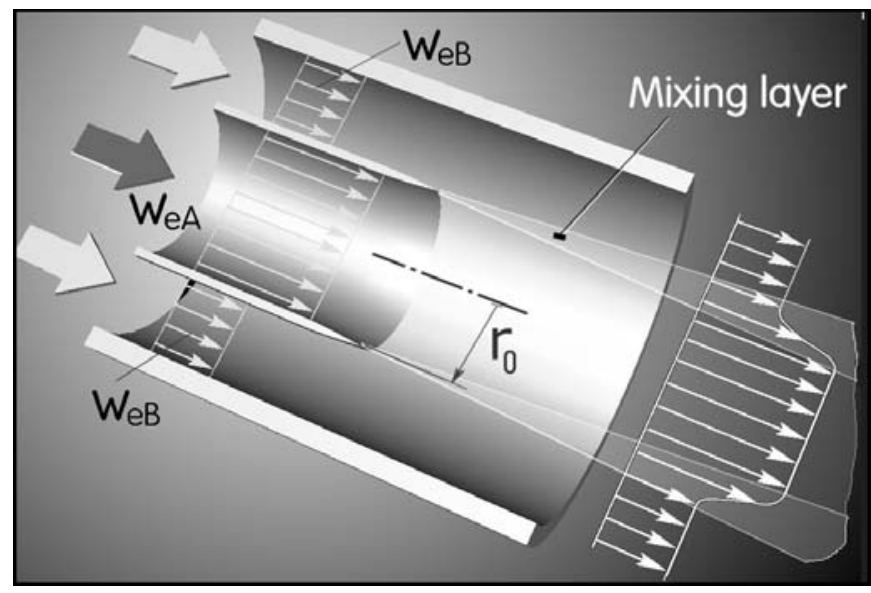

Fig. 2: A transversally curved mixing layer forms when flow of higher velocity $w_{e A}$ leaves the central nozzle and mixes with the outer flow of lower velocity $w_{e B}$. Transversal curvature radius varies with axial distance differently than the layer thickness. This renders an exact similarity of the profiles impossible.

The wakes immediately downstream from the separation wall edge are a complicating factor. Fortunately, they tend to disappear rather fast with increasing streamwise distance. The influence of the wake usually becomes negligible - at least as reflected in the shape of the velocity profiles - at downstream distances equal to a few multiples of the thickness of the boundary layer formed on the separation wall. The similarity approach to the mixing layer is then applicable in the fully developed layer sufficiently far downstream.

The standard solution of the plane mixing layers presented in most textbooks is due to Görtler (1942) which, like the Görtler's solution of the submerged jet mentioned above, is unfortunately based on Prandtl's physically ungrounded "neues Modell". It is the similarity solution in the relative co-ordinates

transverse co-ordinate

$$
\eta_{G}=\sigma_{G} \frac{X_{2}}{X_{1}}
$$

relative velocity

$$
u=\frac{\bar{w}_{1}-w_{e B}}{w_{e A}-w_{e B}}
$$

where the transverse distances $X_{2}$ are measured from the location where $u=0.5, X_{1}$ is the streamwise distance measured from an extrapolated virtual origin, $\bar{w}_{1}$ is the streamwise component of time-mean velocity, and $\sigma_{G}$ is Görtler's proportionality constant in the assumed linear streamwise growth of layer thickness $\delta$

$$
\delta=\frac{X_{1}}{\sigma_{G}}
$$

so that $\eta_{G}=X_{1} / \delta$.

The similarity transformed momentum transport equation with Prandtl's 1942 model of turbulence is an ordinary 
differential equation, which Görtler solved by series expansion. The first term in the power series is dominant. Görtler neglected all remaining terms and found an analytic solution [2] for the first term.

$$
u=\frac{1}{2}\left[1-\operatorname{erf}\left(\sigma_{G} \frac{X_{2}}{X_{1}}\right)\right]
$$

This first-term solution possesses a central symmetry with respect to point $X_{2}=0, u=0.5$, so that $u$ at $-X_{2}$ equals $1-u$ at $X_{2}$. The usual slight deviation from this symmetry, discernible though not prominent in experimental results, is ascribed to the neglected terms.

\subsection{Approximate application to axisymmetric jet flows}

Submerged jets, flows of high importance in engineering applications, gradually develop from the usually nearly uniform velocity profile flow in the nozzle exit [4]. In the earliest stages of development, the flowfield is dominated by the mixing layers between the core flow and the stagnant outer fluid, Fig. 3. Since the developed jets lose their velocity very fast

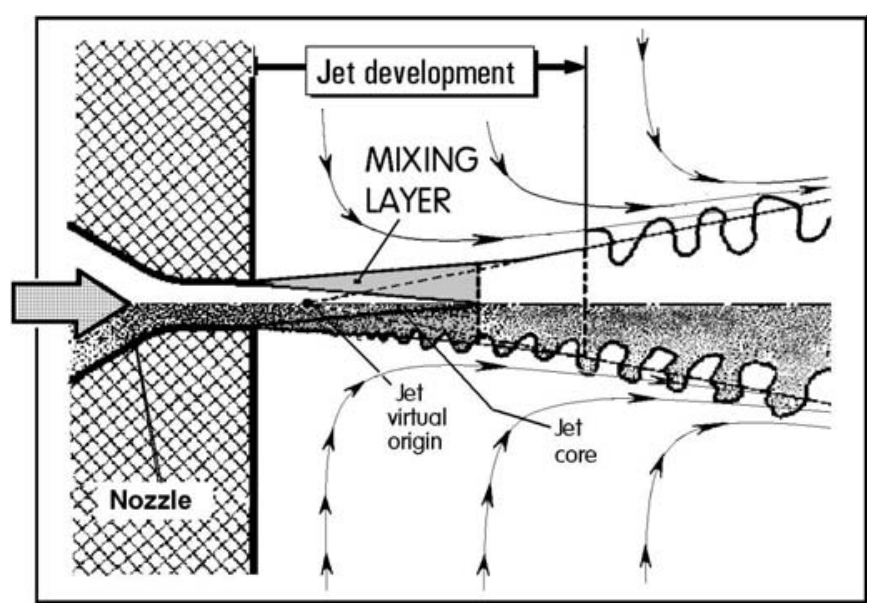

Fig. 3: The mixing layers surrounding the jet core dominate the character of the fluid flow in the developing submerged jet at small downstream distances from the nozzle from which the jet is issuing

with the distance travelled, and hence gradually cease to be able to generate a useful effect, many applications - in particular in fluidics, the technique of controlling fluid flows without the use of moving components in the devices - tend to use only the developing part of the jet. The general rule in fluidic valves, e.g. [19], is to capture the jet while it still contains a significant jet core. As a result, it is of high practical importance to be able to analyse the properties of the mixing layers surrounding the core.

Of course, in the case of a round jet the mixing layer is subject to quite strong transversal curvature. Theoretically, the planar (= infinite curvature radius) conditions are approximated at small downstream distances, where the curvature radius is much larger than the very small layer thickness. Unfortunately, practical considerations limit the applicability of this theoretically sound assumption: a) At small downstream distances the conditions are complicated by the wake of the separation wall edge, which needs a considerable streamwise distance to disappear.

b) In analogy with the conditions in jets [6], the turbulence structure of the mixing layer may be reasonably expected to need a considerable streamwise distance from the separating edge before it develops self-similarity.

In principle, therefore, no similarity solution for the mixing layer at the outer edge of the developing axisymmetric jet should be possible. Fortunately, the effect of curvature is found to be rather weak and experimental evidence shows that the flow may be well approximated by the plane mixing layer solution, indeed with precision sufficient for most engineering applications.

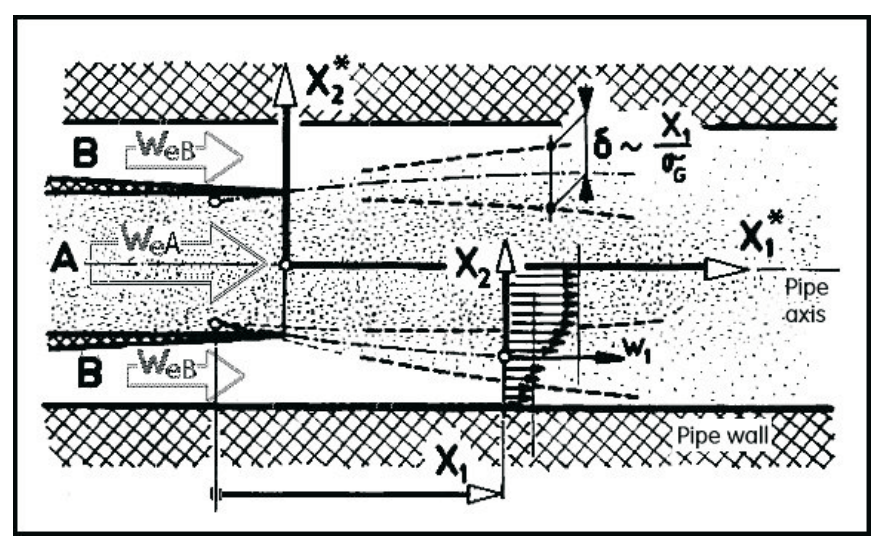

Fig. 4: The mixing layer in a round pipe: faster flow A injected into parallel flow $\mathrm{B}$ of lower velocity $w_{e B}$. Investigations of this layer were performed by the present author in 1978 at CTU in Prague as a part of classified study of radioactive contaminant diffusion towards the pipe wall.

In 1978-79 the present author investigated the mixing layer formed between concurrent flows in a pipe, as shown in Fig. 4, with the faster central flow $w_{e A}>w_{e B}$ injected axially through the central nozzle. Diagrams of the results were used as teaching examples in textbook [2] and the velocity profile diagram was also shown in [15], but because of the classified character of the application in the nuclear industry, the complete results were never properly published. They are of importance in the present context, since they provide an experimental demonstration of several important facts:

- The first of them is the proof of the linear dependence Eq. (3). It is a consequence of very general property of turbulent shear flows. Their thickness grows in convected co-ordinates in proportion to the local velocity scale of turbulence $w_{t}$ (cf. Eq. (5)). For the 1978 experiments this is demonstrated in Fig. 6 by the plot of convention thickness $\delta_{0.8}$ dependence on the streamwise distance $X_{1}^{*}$ from the nozzle exit. The convention definition of the thickness $\delta_{0.8}$ in Fig. 5 was chosen for convenience of processing the experimental data: $\delta_{0.8}$ is the transversal distance between the locations in which the time-mean axial velocity differs from the outer velocity we on each side by $0.1 \Delta w_{e}$, one tenth of the difference between the outer velocities

$\Delta w_{e}=w_{e A}-w_{e B}$.

It should be noted in Fig. 6 that the thickness was not 


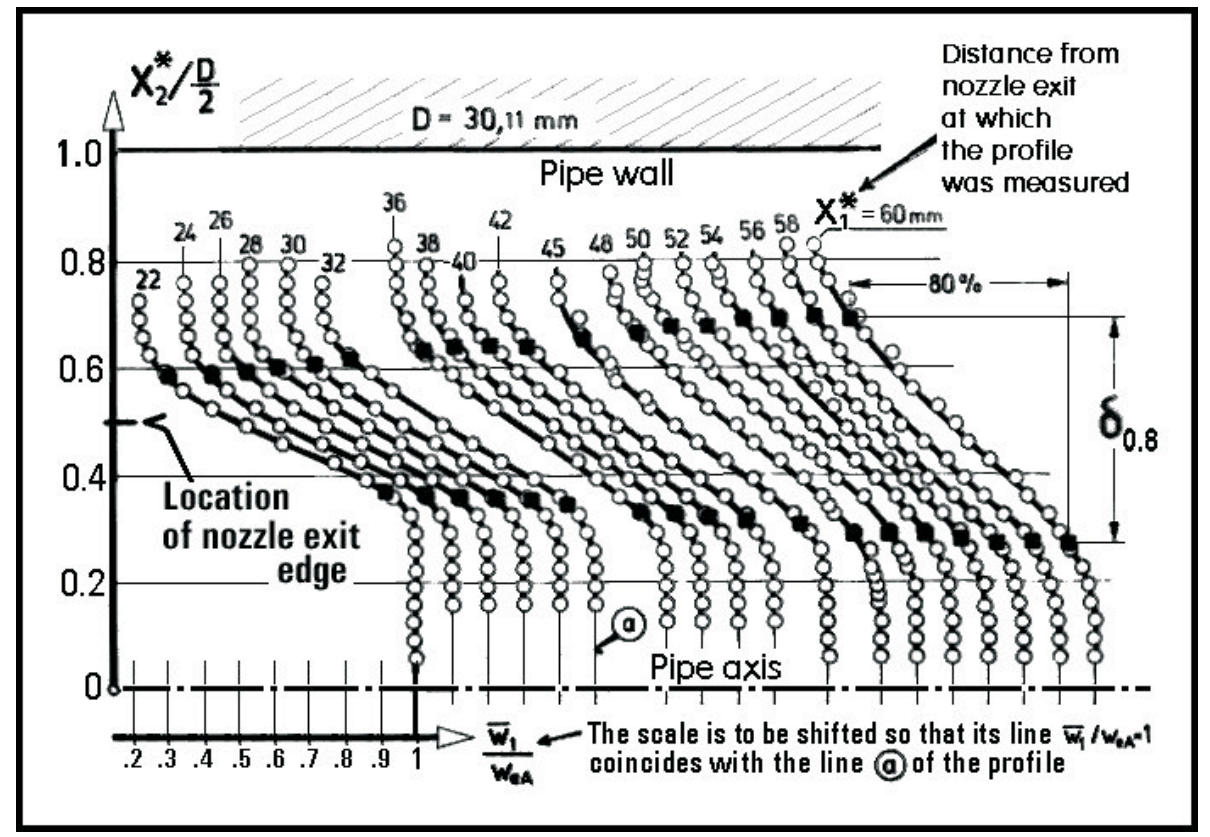

Fig. 5: Unpublished 1978 measurements by a miniature Pitot probe of axial time-mean velocity profiles in the mixing layer inside the pipe of Fig. 3. Convention thickness $\delta_{0.8}$ is defined as the distance between the black symbols, where the velocity differs by $10 \%$ from the velocity outside the layer.

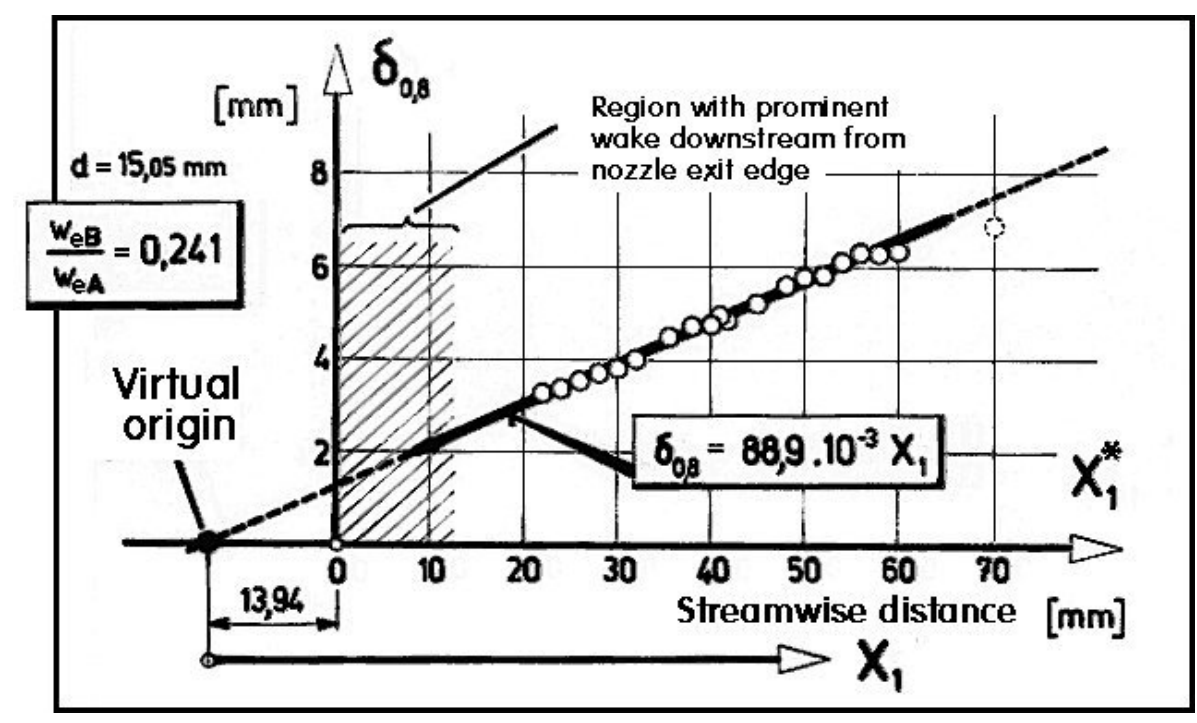

Fig. 6: Streamwise dependence of the convention thickness $\delta_{0.8}$

evaluated in the not fully developed flows at small $X_{1}^{*}$, influenced by the wake downstream from the edge of the nozzle exit. The complicated development necessitated the use of shifted axial co-ordinate $\mathrm{X}_{1}$ measured from the virtual origin. This shift is necessary for changing the axial $\delta_{0.8}$ dependence indicated in Fig. 6 into the linear homogeneous proportionality.

- The second fact of importance is the very small effect of the transversal curvature of the axisymmetric flow, indeed negligible for engineering purposes - despite the curvature radius being comparable here with the layer thickness. This is demonstrated in Fig. 8, which shows four measured velocity profiles, similar to the top example in
Fig. 7, transformed into the similarity co-ordinates Eqs. (1) and (2). The transformation was based on locating the convention edges of the layer according to Fig. 5, and presented in Fig. 9 where it is obvious how near the edges are to axis of the mixing pipe. The next Fig. 10 shows how short is the radius $r$ of the transversal curvature relative to the layer thickness $\delta$. The table in Fig. 10 shows how the ratio $r / \delta$ decreases with the downstream distance $\mathrm{X}_{1}$. Despite this fact, the transformed profiles measured at different $\mathrm{X}_{1}$ are in Fig. 8 practically identical. Their mutual differences are smaller than the experimental scatter (which is remarkably small, considering the stochastic nature of the turbulent flow and the small dimensions of the experiment - pipe internal diameter a mere $30.11 \mathrm{~mm}$ ). As 


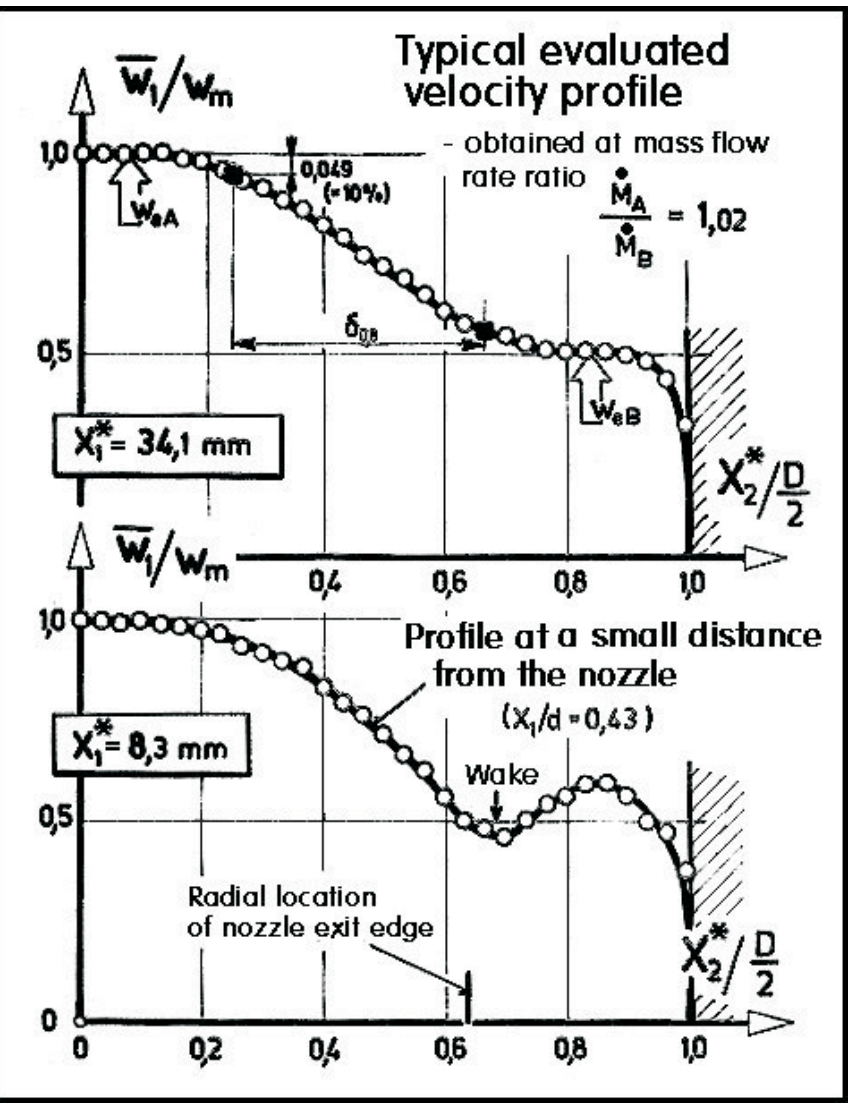

Fig. 7: The character of measured velocity profiles in the pipe. If there was a prominent wake (as in the example at downstream distance $8.3 \mathrm{~mm}$ ), the data were not included into the comparison with the similarity solution, Fig. 8. was usual at that time, the present author compared the measured profiles with the Görtler solution, Eq. (4). The good agreement provides an explanation for the continuing popularity of this solution, despite its lack of a sound theoretical foundation.

\section{Hierarchy of three turbulence models}

\subsection{Isotropic models}

The models discussed here assume isotropic turbulence. Of course, large eddies in a mixing layer generally exhibit a preferred spatial orientation (with axes parallel to the edge of the dividing wall). Their anisotropy, however, does not warrant the use of more complex anisotropic models. Most influential in the turbulent momentum transport are smaller vortices, of characteristic size roughly twenty times smaller than the local layer thickness. Their orientation is near to chaotic, and there is hardly any orientation preference.

Isotropic turbulence is fully specified by a single parameter - the turbulent viscosity $v_{t}$. This is a product of two factors (using the same symbols as in earlier publications [2, 3, 5, and 6]):

$$
v_{t}=w_{t} \ell,
$$

where $\ell[\mathrm{m}]$ is turbulence length scale - size of vortices most effective in turbulent momentum transport), and $w_{t}[\mathrm{~m} / \mathrm{s}]$ is the velocity scale of turbulence.

\section{Algebraic model}

The turbulent viscosity $v_{t}$ is evaluated solving algebraic equations based on two hypotheses:

$$
\text { 1) Prandtl hypothesis: } \quad w_{t}=\frac{\partial \bar{w}_{1}}{\partial X_{2}} \ell
$$

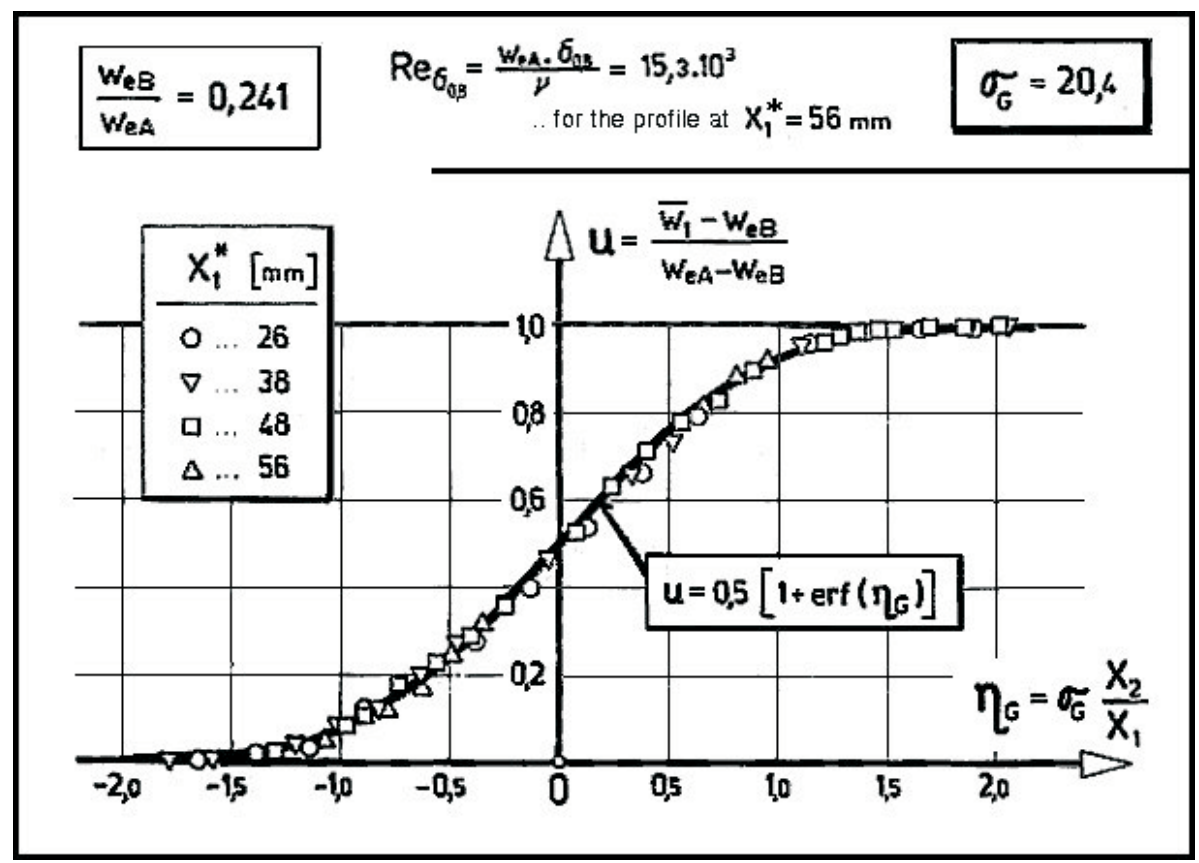

Fig. 8: Velocity profiles of the mixing layer, Figs. 3 and 4, plotted in the similarity co-ordinates and compared with the first-term Görtler solution Eq. (4). No significant systematic effect of the transverse curvature is discernible. 


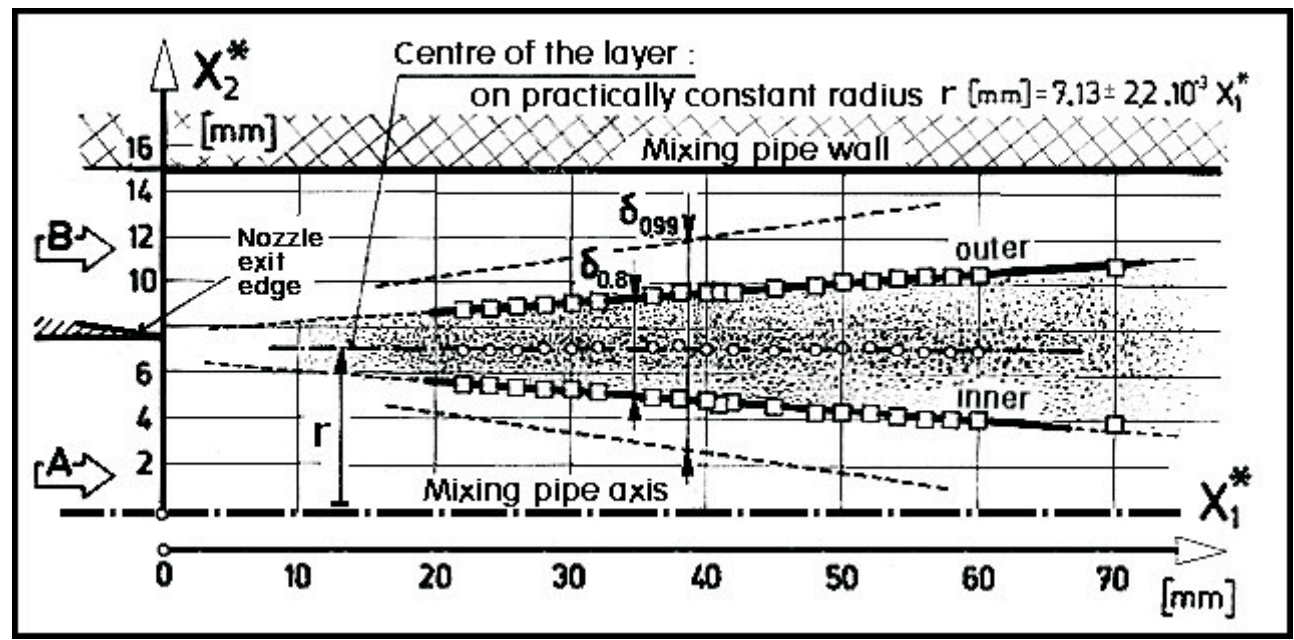

Fig. 9: Definition of the convention edges of the mixing layer. The layer is quite thin and long (note the compression of the axial scale), its radius of transversal curvature is quite small.

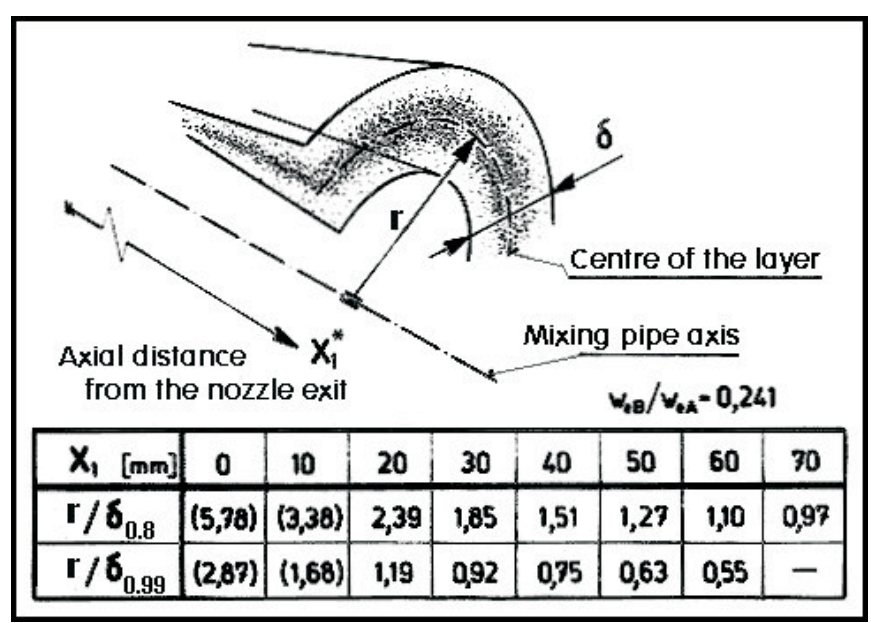

Fig. 10: The negligible effect in Fig. 4 of the transversal curvature is quite surprising, considering the fact that the curvature radius is comparable with the layer thickness $\delta$.

turbulent eddies are set into rotation by the velocity difference between their extreme ends, due to the local transverse gradient $\frac{\partial \bar{w}_{1}}{\partial X_{2}}$ of streamwise velocity $\bar{w}_{1}$.

2) Tollmien hypothesis:

$$
\ell=k \delta
$$

eddy size is constant across each transversal section of the layer, its magnitude increases with the thickness $\delta$ of the layer.

\section{1-Equation model}

The turbulent viscosity $v_{t}$ is evaluated solving the transport equation for specific energy of turbulent fluctuation $e_{f}$ and making use of two relations:
1) Prandtl-Kolmogorov expression: $w_{t}=c_{v} \sqrt{e_{f}}$
2) Tollmien hypothesis:
$\ell=k \delta$

\section{2-Equation model}

The turbulent viscosity $v_{t}$ is evaluated solving two transport equations - one for specific energy of turbulent fluctuation $e_{f}$ and the other for the turbulence dissipation rate $\in$. The resultant spatial distributions are used in the relations:
1) Prandtl-Kolmogorov expression: $w_{t}=c_{v} \sqrt{e_{f}}$

2) Expression due to

\subsection{Similarity transformation with the algebraic model}

Using the notation from textbook [2], the mixing layer flowfield is described by Prandtl's thin shear flow equation for spatial distribution of time-mean axial velocity $\bar{w}_{1}$

$\frac{\partial \bar{w}_{1}}{\partial X_{1}} \bar{w}_{1}+\frac{\partial \bar{w}_{1}}{\partial X_{2}} \bar{w}_{2}-\frac{\partial^{2} \bar{w}_{1}}{\partial X_{2}^{2}} v_{t}-\frac{\partial \bar{w}_{1}}{\partial X_{2}}\left(\frac{\partial v_{t}}{\partial X_{2}}\right)=0$,

the algebraic model Eqs. (5), (6), and (15) expresses the turbulent viscosity $v_{t}$ as

$$
v_{t}=\frac{\partial \bar{w}_{1}}{\partial X_{2}}(k s)^{2} X_{1}^{2},
$$

and its transverse gradient

$$
\frac{\partial v_{t}}{\partial X_{2}}=\frac{\partial^{2} \bar{w}_{1}}{\partial X_{2}^{2}}(k s)^{2} X_{1}^{2}
$$

Following closely the transformation procedure described in [2] and [3], where there are more detailed explanations, Eq. (12) with inserted expressions Eqs. (13) and (14) is transformed using the definition of the relative transverse co-ordinate according to [3]

$$
\eta_{A L G}=\frac{X_{2}}{\sqrt[3]{2(k s)^{2}} X_{1}},
$$

where $k$ is the proportionality constant from Eq.(7) and $s$ is the proportionality factor in the linear streamwise growth relation for layer thickness $\delta$ :

$$
\delta=s X_{1}
$$

The definition Eq. (15) differs from the original one used in [3] by the added index $A L G$ to remove possible confusions with several other similarity co-ordinate definitions in the present paper. It should be also noted that the thickness Eq. (16) is different from Görtler's $\delta$ in Eq. (3) because of the different definitions in Eqs.(1) and (15). From Eqs. (7) and (16), 


$$
\ell=k s X_{1}
$$

To evade repeated checking of the conformity of solutions with the mass conservation condition, in the present two-dimensional case it is useful to re-write Eq. (12) in terms of stream function $\Psi$, related by means of eq.(15) to transformed relative stream function $f$

$$
\Psi=f \sqrt[3]{2(k s)^{2}} X_{1} \Delta w_{e} .
$$

The transformation into the similarity form follows closely the analogous approach for submerged plane jets in [3] - the dissimilarity in the present case is in the constant velocity difference

$$
\Delta w_{e}=w_{e A}-w_{e B}
$$

replacing the maximum velocity in the velocity profiles of the jets in [3], which varies in streamwise direction. Transformed velocities and their derivatives are:

$$
\begin{aligned}
& \bar{w}_{1}=\frac{\mathrm{d} f}{\mathrm{~d} \eta} \Delta w_{e}, \\
& \bar{w}_{2}=-f \sqrt[3]{2(k s)^{2}} \Delta w_{e}+\frac{\mathrm{d} f}{\mathrm{~d} \eta} \frac{X_{2} \Delta w_{e}}{X_{1}}, \\
& \frac{\partial \bar{w}_{1}}{\partial X_{1}}=-\frac{\mathrm{d}^{2} f}{\mathrm{~d} \eta^{2}} \frac{X_{2} \Delta w_{e}}{\sqrt[3]{2(k s)^{2}} X_{1}^{2}}, \\
& \frac{\partial \bar{w}_{1}}{\partial X_{2}}=\frac{\mathrm{d}^{2} f}{\mathrm{~d} \eta^{2}} \frac{\Delta w_{e}}{\sqrt[3]{2(k s)^{2}} X_{1}}, \\
& \frac{\partial^{2} \bar{w}_{1}}{\partial X_{2}^{2}}=\frac{\mathrm{d}^{3} f}{\mathrm{~d} \eta^{3}} \frac{\Delta w_{e}}{\sqrt[3]{4(k s)^{4}} X_{1}^{2}} .
\end{aligned}
$$

Inserting these expressions into the momentum transport equation (12) converts it into

$\frac{\mathrm{d}^{2} f}{\mathrm{~d} \eta^{2}}\left(\frac{\mathrm{d} f}{\mathrm{~d} \eta}\right) \frac{X_{2} \Delta w_{e}^{2}}{\sqrt[3]{2(k s)^{2}} X_{1}^{2}}-\frac{\mathrm{d}^{2} f}{\mathrm{~d} \eta^{2}}\left(\frac{\mathrm{d} f}{\mathrm{~d} \eta}\right) \frac{X_{2} \Delta w_{e}^{2}}{\sqrt[3]{2(k s)^{2}} X_{1}^{2}}-$

$\frac{\mathrm{d}^{2} f}{\mathrm{~d} \eta^{2}} f \frac{\Delta w_{e}^{2}}{X_{1}}-2 \frac{\mathrm{d}^{3} f}{\mathrm{~d} \eta^{3}}\left(\frac{\mathrm{d}^{2} f}{\mathrm{~d} \eta^{2}}\right) \frac{\Delta w_{e}^{2}}{2 X_{1}}=0$

Mutual cancellation of the first and second terms and division by $\frac{\Delta w_{e}^{2}}{X_{1}}$ results in the final form of the similarity transformed governing equations

$$
\frac{\mathrm{d}^{3} f}{\mathrm{~d} \eta^{3}}+f=0 \text { or } \frac{\mathrm{d}^{2} f}{\mathrm{~d} \eta^{2}}=0
$$

\subsection{Similarity transformation with the 1-Equation model}

Again following closely the details of the derivation for the jet in [3], the Prandtl equation (12) is transformed using the definition of the relative transverse co-ordinate

$$
\eta_{1 E Q}=\frac{X_{2}}{\sqrt{k s c_{v} X_{1}}}
$$

where $c_{v}$ is the proportionality constant from Eq. (8), known in equilibrium turbulence to possess [2] the value $c_{v}=0.548$, while $k$ and $s$ are as in Eq. (16) and (17). Again, this definition differs from the original in [3] by an added index - here the index $1 E Q$ to discriminate the variable introduced in Eq. (21) from the different definitions Eqs. (1) and (15). This turbulence model requires that simultaneously with the momentum transport equation is solved also the transport equation for the specific energy of turbulent fluctuations $e_{f}$

$\frac{\partial e_{f}}{\partial X_{1}} \bar{w}_{1}+\frac{\partial e_{f}}{\partial X_{2}} \bar{w}_{2}-\frac{\partial^{2} e_{f}}{\partial X_{2}^{2}} v_{t}-\frac{\partial e_{f}}{\partial X_{2}}\left(\frac{\partial v_{t}}{\partial X_{2}}\right)=\mathcal{P}-\epsilon$,

where $P$ is the turbulence production rate [2]

$$
\mathcal{P}=\left(\bar{w}_{1} \nabla_{2}\right)^{2} v_{t}
$$

and $\in$ is the dissipation rate

$$
\in=c_{z} \frac{\sqrt{e_{f}^{3}}}{k s X_{1}}
$$

The coefficient of the gradient transport rate in Eq. (22) is $v_{t}$ - the same as in Eq. (12), i.e. with the Prandtl number for gradient transport of fluctuations $\operatorname{Pr}_{\text {tef. }}=1.0$. This is a standard assumption, equivalent to the expectation of all quantities being transported by turbulent motions equally. As presented in [2], the standard value of the model constant in Eq. (24) is $c_{z}=0.164$.

The transformation into the similarity form, again following closely the analogous approach for submerged plane jets in [3], differs from the jet case by the absence of streamwise variation of the velocity reference in the streamwise direction. The reference value used is the constant value from Eq. (19). Transformation of Eq. (22) requires a suitably defined relative value of the specific energy of turbulent fluctuations: using the velocity reference (19) it is

$$
\varepsilon=\frac{e_{f}}{\Delta w_{e}^{2}} .
$$

For the same reasons as in the algebraic model case, it is useful to re-write Eq. (12) in terms of stream function $\Psi$, which is now related by the definition Eq. (21) to the transformed relative stream function $f$

$$
\Psi=f \sqrt{k s c_{v}} X_{1} \Delta w_{e}
$$

The 1-equation model, Eqs. (8), (9), together with the usual Eqs. (5) and (16) leads to the following expression for the turbulent viscosity, using the relative value $\varepsilon$ Eq. (25) of the fluctuation energy

$$
v_{t}=\sqrt{\varepsilon} k s c_{v} X_{1} \Delta w_{e} .
$$

The transverse gradient of the turbulent viscosity is expressed by means of $\varepsilon$

$$
\frac{\partial v_{t}}{\partial X_{2}}=\frac{1}{2} \frac{\varepsilon \nabla}{\sqrt{\varepsilon}} \sqrt{k s c_{v}} \Delta w_{e} .
$$

The transformed velocities and their derivatives are $\bar{w}_{1}=\frac{\mathrm{d} f}{\mathrm{~d} \eta} \Delta w_{e}$,

$\bar{w}_{2}=-f \sqrt{k s c_{v}} \Delta w_{e}+\frac{\mathrm{d} f}{\mathrm{~d} \eta} \frac{X_{2} \Delta w_{e}}{X_{1}}$,

$\frac{\partial \bar{w}_{1}}{\partial X_{1}}=-\frac{\mathrm{d}^{2} f}{\mathrm{~d} \eta^{2}} \frac{X_{2} \Delta w_{e}}{\sqrt{k s c_{v}} X_{1}^{2}}$, 
$\frac{\partial \bar{w}_{1}}{\partial X_{2}}=\frac{\mathrm{d}^{2} f}{\mathrm{~d} \eta^{2}} \frac{\Delta w_{e}}{\sqrt{k s c_{v}} X_{1}}$,

$\frac{\partial^{2} \bar{w}_{1}}{\partial X_{2}^{2}}=\frac{\mathrm{d}^{3} f}{\mathrm{~d} \eta^{3}} \frac{\Delta w_{e}}{k s c_{v} X_{1}^{2}}$,

and the transformed terms in Eq. (22) are

$$
\begin{gathered}
\frac{\partial e_{f}}{\partial X_{1}}=-\frac{\mathrm{d} \varepsilon}{\mathrm{d} \eta} \frac{X_{2} \Delta w_{e}^{2}}{\sqrt{k s c_{v}} X_{1}^{2}}, \\
\frac{\partial e_{f}}{\partial X_{2}}=-\frac{\mathrm{d} \varepsilon}{\mathrm{d} \eta} \frac{\Delta w_{e}^{2}}{\sqrt{k s c_{v}} X_{1}}, \\
\frac{\partial^{2} e_{f}}{\partial X_{2}^{2}}=\frac{\mathrm{d}^{2} \varepsilon}{\mathrm{d} \eta^{2}} \frac{\Delta w_{e}^{2}}{k s c_{v} X_{1}^{2}} .
\end{gathered}
$$

Inserting these expressions into the momentum transport Eq. (12), converts it after mutual cancellation of the first and second terms, and division by $\frac{\Delta w_{e}^{2}}{X_{1} \sqrt{\varepsilon}}$ into the final form

$2 \frac{\mathrm{d}^{3} f}{\mathrm{~d} \eta^{3}} \varepsilon+\frac{\mathrm{d}^{2} f}{\mathrm{~d} \eta^{2}}\left(\frac{\mathrm{d} \varepsilon}{\mathrm{d} \eta}\right)+2 \frac{\mathrm{d}^{2} f}{\mathrm{~d} \eta^{2}} f \sqrt{\varepsilon}=0$

An analogous procedure applied to Eq. (22) results in

$2 \frac{\mathrm{d}^{2} \varepsilon}{\mathrm{d} \eta^{2}} \varepsilon+2 \frac{\mathrm{d} \varepsilon}{\mathrm{d} \eta} f \sqrt{\varepsilon}+\left(\frac{\mathrm{d} \varepsilon}{\mathrm{d} \eta}\right)^{2}+2\left(\frac{\mathrm{d}^{2} f}{\mathrm{~d} \eta^{2}}\right)^{2} \varepsilon-2 \varepsilon^{2} \frac{c_{z}}{k s}=0$.

The solution of these simultaneous equations is complicated by the unknown parameter $\frac{c_{z}}{k s}$. An analogous problem arose in [3], where it was solved by using experimental data about the magnitude of the thickness growth factor $s$.

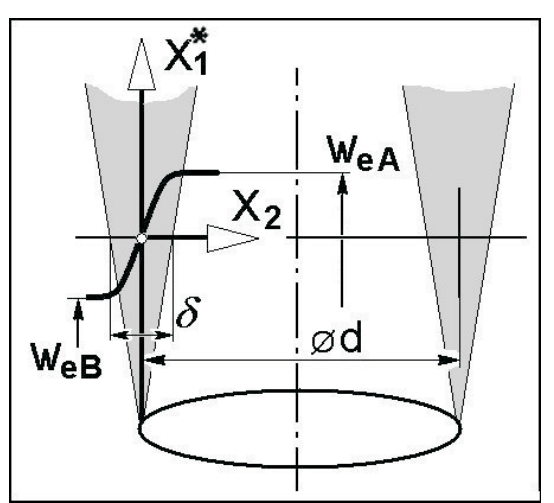

Fig. 11: Schematic representation of the investigated mixing layers surrounding the core of a round jet with a zero axial component of the outer velocity $w_{e B}$.

\section{Experiment: PIV measurements in a developing jet}

The experimental facility used for generation of the investigated developing jets was designed by the present author for investigations of helicity transport in swirling jets [15]. It was tested in the initial stages of the project without imparting the swirl to the jet [7] and it is the data from these preliminary tests which were used for the present investigations. The round nozzle exit was of $d=32 \mathrm{~mm}$ diameter. The working fluid was air at atmospheric conditions, seeded upstream from the nozzle with water particles of $0.19 \mathrm{~mm}$ mean diameter. The Reynolds number evaluated for the nozzle exit conditions

$$
\operatorname{Re}_{d}=4.54 \times 10^{3}
$$

though not very high, was sufficient for reasonably well developed turbulence in the mixing regions of the jet. Schematic representation of the investigated flow and definition of

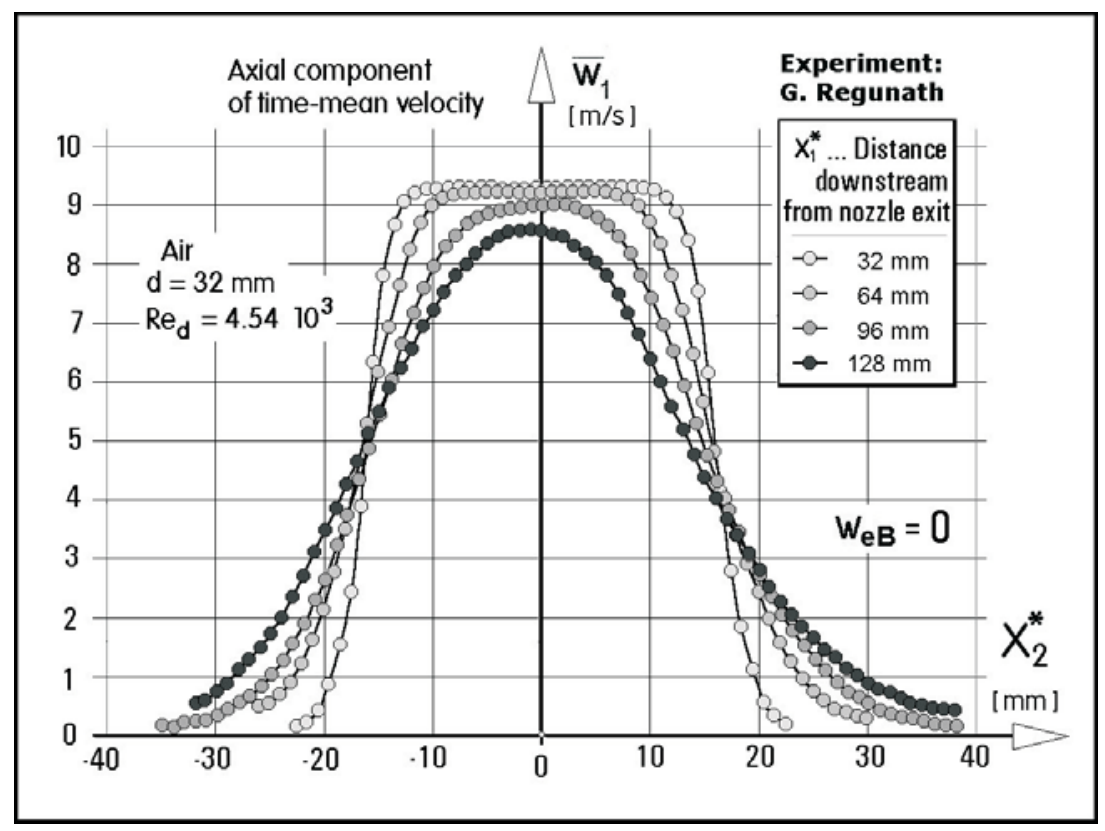

Fig. 12: Velocity profiles obtained by PIV measurements in an air jet (data obtained in collaboration with G. Regunath). Only three profiles nearest to the exit were used for the comparison with the similarity solutions. The slight asymmetry of the profiles is hardly avoidable in practical flows. 


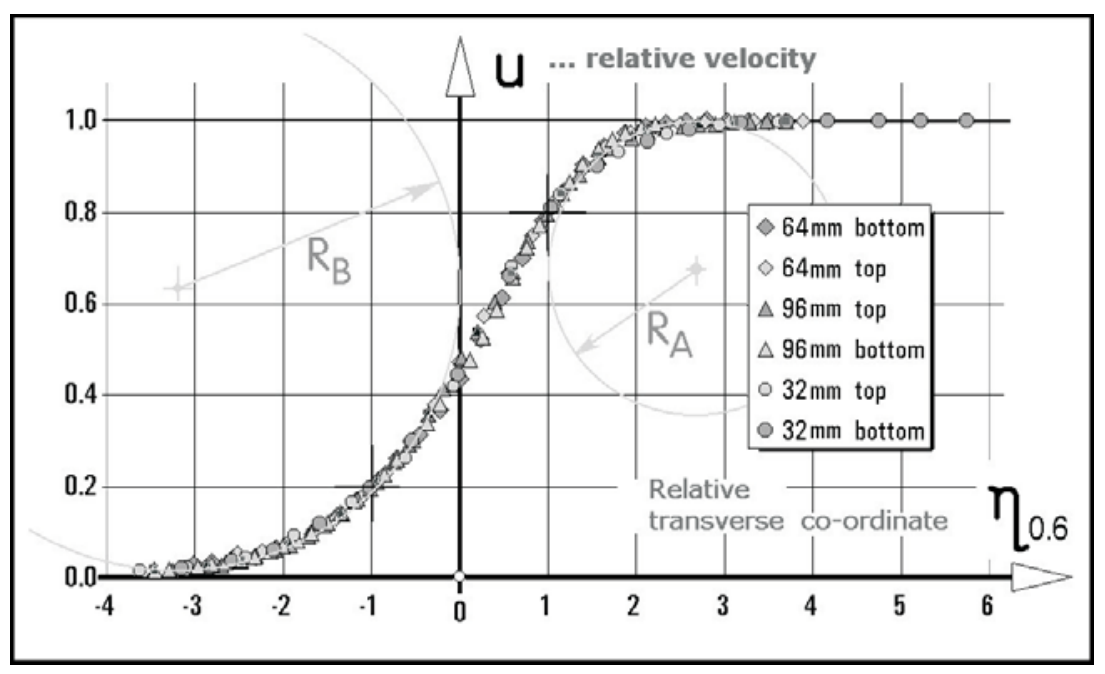

Fig. 13: Velocity data evaluated from Fig. 12 and re-plotted in similarity variables. No significant effect of transversal curvature (which increases with $X_{1}^{*}$ ) was found. The different osculation radii $\mathrm{R}_{\mathrm{A}}$ and $\mathrm{R}_{\mathrm{B}}$ indicate lack of central symmetry, a feature of the usually presented Görtler's solution in its first-term form Eq.(4).

the used co-ordinate axes is presented in Fig. 11. The axial component of the external velocity was practically zero, $w_{e B}=0$.

The Particle Image Velocimetry (PIV) system, supplied by Oxford Lasers Inc., was used for velocity measurement. The flowfield was illuminated by a twin pulse Nd:YAG laser with wavelength of the generated light $532 \mathrm{~nm}$. The laser emits two laser pulses of 5 ns duration, with energy of $50 \mathrm{~mJ}$ per pulse. In the present case, the time delay between the two laser pulses was set to $18 \mu \mathrm{s}-40 \mu \mathrm{s}$ to ensure that the particle-image displacement is about one-quarter of the interrogation domain. The light sheet, positioned in the meridian plane (passing through the jet axis) was less that $2 \mathrm{~mm}$ in thickness. The intensity of the light reflected from the tracer particles is recorded separately by two synchronised digital CCD cameras (PCO Sensicam). The data were processed by VidPIV software package, also supplied by Oxford Lasers Inc.

The data were collected under present author's supervision by Gavita Regunath. They consist of sets of time-mean velocity values obtained at constant $1 \mathrm{~mm}$ steps along transverse lines perpendicular to the nozzle axis. Of them, three velocity profiles located at $32 \mathrm{~mm}$ (- one nozzle diameter), $64 \mathrm{~mm}$ (- two diameters) and $96 \mathrm{~mm}(-3 d)$, as shown in Fig. 12 were chosen as potentially suitable for validation of the similarity solutions. Previous positive experience with an analogous evaluation of the axisymmetric mixing layer suggested that a validation based on comparison with planar mixing layer solutions is worthwhile.

The similarity co-ordinate for the velocities used for presentation of the data in Fig. 13 is defined by Eq. (2) - it is the same as for Görtler solution. However, the transformed distance co-ordinate is neither of those defined above - neither the Görtler's Eq. (1) nor Eqs. (15), (20), and (33) of the better models - because of the absence at the initial stage of data processing of knowledge about the quantities used in these later definitions. The problem was circumvented by using yet another dimensionless co-ordinate $\eta_{0.6}$, related to the convention thickness $\delta_{0.6}$, analogous to $\delta_{0.8}$ specified in Figs. 4 and 6 - here, however, $\delta_{0.6}$ is the distance between the loca-

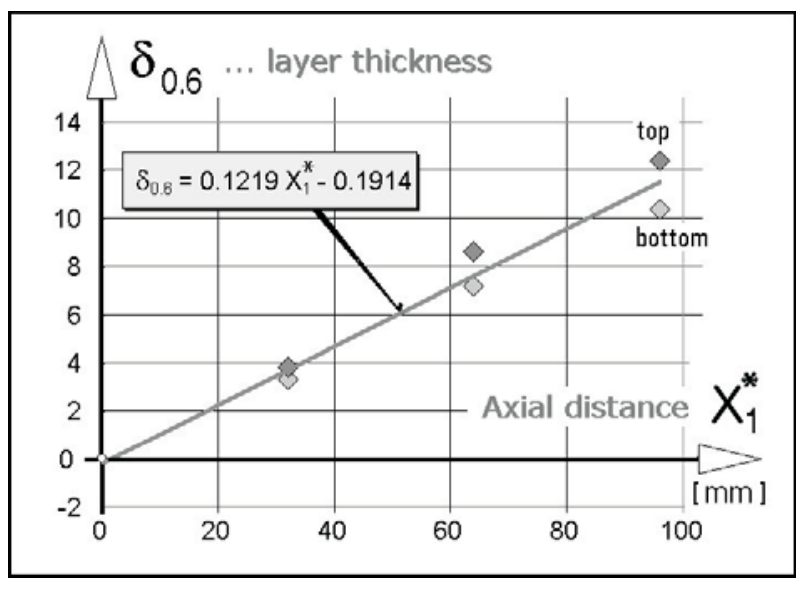

Fig. 14: Convention thickness $\delta_{0.6}$ dependence on the downstream distance $X_{1}^{*}$. Resultant least-squares expression is fitted through all experimental data as a mean between the not perfectly symmetric top and bottom sides of the jet.

tions in which the velocity $\bar{w}_{1}$ differs from the outer velocity $w_{e}$ on each side by $0.2 \Delta w_{e}$. Fig. 14 presents an evaluation of the growth factor $s_{0.6}$ in $\delta_{0.6}=s_{0.6} X_{1}$, (a version of Eq. (16)) from the experimental data.

\section{Solutions of the transformed equations}

\subsection{Algebraic model}

It is useful to introduce auxiliary variables, identical to those used in [3] and [5]:

$u=\frac{\bar{w}_{1}}{\Delta w_{e}}=\frac{\mathrm{d} f}{\mathrm{~d} \eta} \quad$ dimensionless axial time-mean velocity,

and

$g$

dimensionless transversal gradient of the axial time-mean velocity. 
The two ordinary differential equations Eq. (19) describing the mixing layer flow in the transformed co-ordinates are then decomposed into the set of three first-order equations

$$
\left.\begin{array}{l}
\frac{\mathrm{d} f}{\mathrm{~d} \eta}=u \\
\frac{\mathrm{d} u}{\mathrm{~d} \eta}=g \\
\frac{\mathrm{d} g}{\mathrm{~d} \eta}=-f \text { or } g=0
\end{array}\right\}
$$

of which the last one has two alternatives. The meaning and necessity of this somewhat unusual feature is discussed below.

The next part of the problem is to determine the set of boundary conditions at a point which is chosen as the starting point of the integration. There is the trivial set of zero boundary conditions $f=u=g=0$ outside the layer, but trying to start the integration from this boundary is useless, the values would simply remain zero. There is, unfortunately, no other point across the layer in which all the three values are known. The best choice seemed to be to select as the starting point the location at which there is $\frac{\mathrm{d} g}{\mathrm{~d} \eta}=0$, since for the algebraic model this means also $f=0$. It is then necessary to find there the proper starting values for $u$ and $g$. The location must be near to the centre of symmetry point $u=0.5$ of the Görtler solution, because the velocity profile $u=f(\eta)$ there may be roughly approximated by a sloping straight line, for which the distribution of the gradient $g=f(\eta)$ is a second-order parabola with its vertex $\frac{\mathrm{d} g}{\mathrm{~d} \eta}=0$ just at this location. Nevertheless, the obvious asymmetry as seen in the experimental data in Fig. 11 requires the location of the maximum gradient $g$ - and the corresponding starting point $\eta_{A L G}=0$ of the integration -

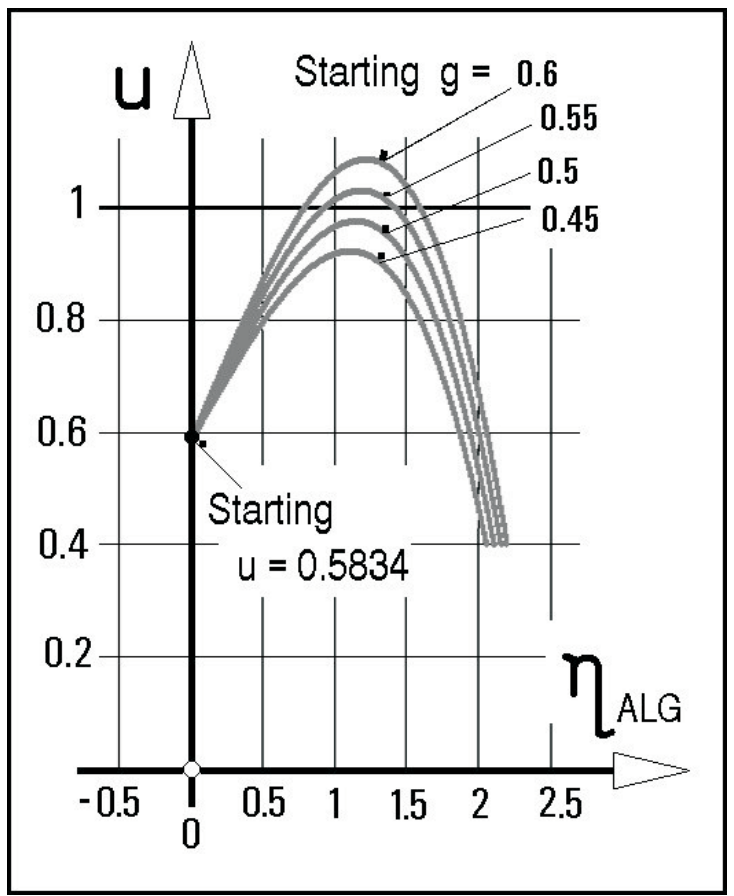

Fig. 15: Solutions for the dimensionless velocity $u$ obtained by numerical integration of the set of equations $\mathrm{d} f / \mathrm{d} \eta=u$, $\mathrm{d} u / \mathrm{d} \eta=g$, and $\mathrm{d} g / \mathrm{d} \eta=-f$ in the direction of the positive transversal similarity co-ordinate $\eta_{A L G}$. to be slightly to the right-hand side, at a slightly higher value of $u$. Cut-and-try manipulation of the starting conditions finally led to satisfactory starting values found at $g=0.523$ and $u=0.5834$. Some exact optimisation algorithm could be applied but this was considered unnecessary since the algebraic model cannot be expected anyway to provide a really good correspondence with real flows and is discussed here as a mere starting stage for later more sophisticated solutions.

With the above starting values, the velocity profiles obtained by the Runge-Kutta integration procedure consists of two parabolic arcs. Fig. 15 presents the integral curves at positive $\eta_{A L G}$ values for several starting values of the dimensionless velocity gradient $g$. With the proper values for both $u$ and $g$ there is a maximum $u=1$ at $\eta_{A L G}=1.166581$. On the negative $\eta_{A L G}$ side there is a minimum $u=0$ at $\eta_{A L G}=-1.846694$. The explanation for the perhaps somewhat strange existence of two alternatives for the last equation in Eq. (32) becomes apparent in Fig. 16: the other equation $\frac{\mathrm{d} u}{\mathrm{~d} \eta}=g=0 \quad$ provides the smooth continuation beyond the extremes, where $\frac{\mathrm{d} g}{\mathrm{~d} \eta}=-f$ would lead to a physically wrong decrease in the velocity. Qualitatively similar, though numerically different, is the situation on the left-hand side of negative distances $\eta_{A L G}$. The complete solution, not only for the velocity profile $u=f(\eta)$ but also for the profiles of the other transformed quantities of interest for the algebraic model is shown in Fig. 16.

The algebraic solution for relative velocity $u$ shown in Fig. 17 reaches the value $u=0.8$ at point A where $\eta_{A L G}=0.4304798$. On the other side, the value $u=0.2$ at point $\mathrm{B}$ is reached at $\eta_{A L G}=-0.8160231$. These two values provide the information needed for conversions between $\eta_{0.6}$ used to process the experimental data and the present co-or-

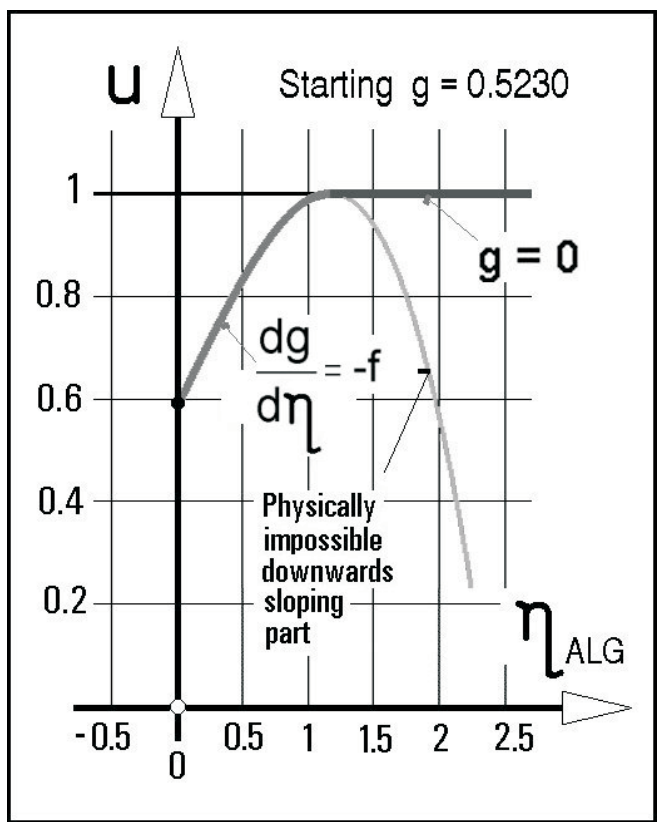

Fig. 16: With $g=0.523$ the solution curve just reaches $u=1$ at the vertex of the parabola. Thereafter, the physically unacceptable decrease is avoided by switching from the solved equation $\mathrm{d} g / \mathrm{d} \eta=-f$ to the other alternative $g=0$. 


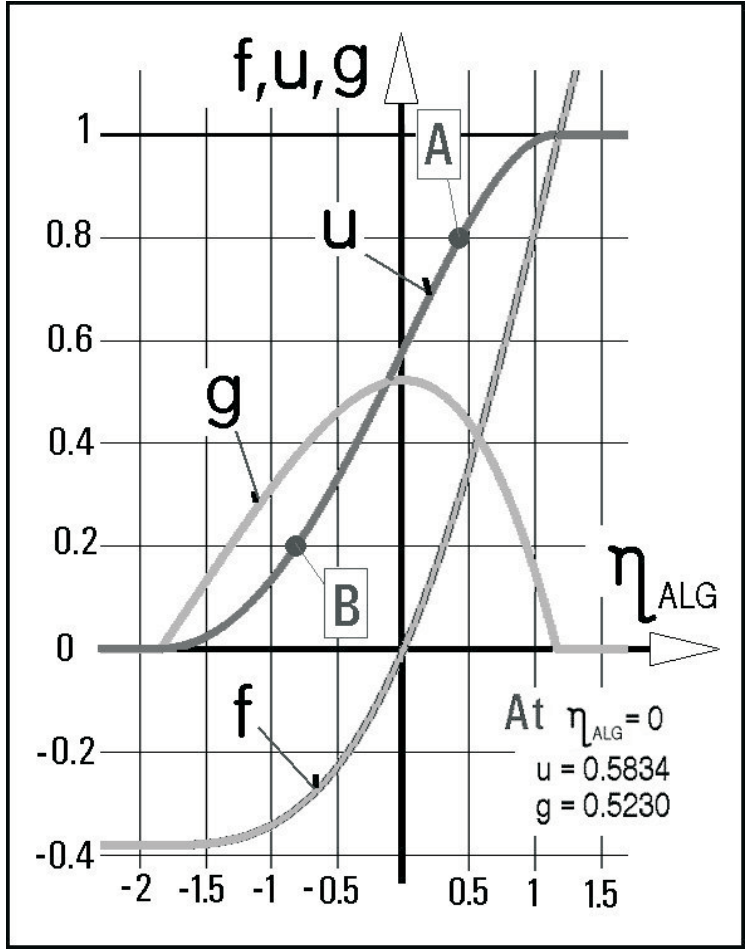

Fig. 17: Complete solution of the planar mixing layer profiles of relative velocity $u$, its gradient $g$, and the relative stream function $f$ - using the algebraic model of turbulence. Eq. (42) integrated using the Runge-Kutta algorithm and the indicated boundary condition values at $\eta_{A L G}=0$.

dinate $\eta_{A L G}$. Note that because of the choice of the starting point for the integration, the positions have to be shifted horizontally by -0.192772 . When expressed in terms of $\eta_{0.6}$, the values indicating the position are larger and have to be multiplied by 0.62325145 (= one half of the distance between points A and B) to obtain the distance in terms of $\eta_{A L G}$.

This conversion provides an opportunity for comparing the similarity solution using the algebraic model with the experimental data. It should perhaps be stressed that these data are by no means an infallible reference. Besides the obvious possibility of the influence of transverse curvature, it should also be kept in mind that they were taken at Reynolds number (cf. Eq. (31)) too small to secure the fully developed turbulence which is assumed in the model. Also the development distance downstream from nozzle exit edge is here almost certainly too short for the turbulence achieving full similarity. There were also inevitable inaccuracies in the experiment, as is visible from e.g. the imperfect symmetry in Fig. 12.

Considering all such negative factors, the agreement of the dimensionless velocities obtained from the similarity solution and from the experiment as presented in Fig. 18 must be described as very good. Indeed, the solution is perhaps sufficiently accurate for most practical engineering applications.

Despite the extreme simplicity of the solved equations (cf. Eq. (32)), this similarity solution - in contrast to the central symmetry of the correlations used earlier, as shown e.g. in Fig. 8 - even results in profiles which are asymmetric, exhibiting better agreement with reality. Though it must be admitted that the radii of the osculation circles (cf. Fig. 13) are not rendered particularly well, they are at least not the same for positive and for negative $\eta_{A L G}$ and reflect the fact that the radius $\mathrm{RB}$ on the negative $\eta_{A L G}$ side is larger.

As could be expected, the agreement is less perfect for the values of the first derivative in Fig. 19 and it is even worse for the second derivative of the velocity in Fig. 20. The derivatives were evaluated for the experimental data using the 5-point numerical differentiation scheme (which provides some smoothing effect).

Of particular importance is the comparison presented in Fig. 20. Considering all the possible sources of inaccuracies and discrepancies, there is actually a very good agreement between the similarity solution and the experiment in the central part of the diagram, roughly between $\eta_{A L G}=-0.5$ and $\eta_{A L G}=0.5$. Beyond these limits, the solution and data diverge quite widely. Since the algebraic model is a reasonable description of transport-less turbulence, the conclusion from Fig. 20 is that the turbulence is very probably in near equilib-

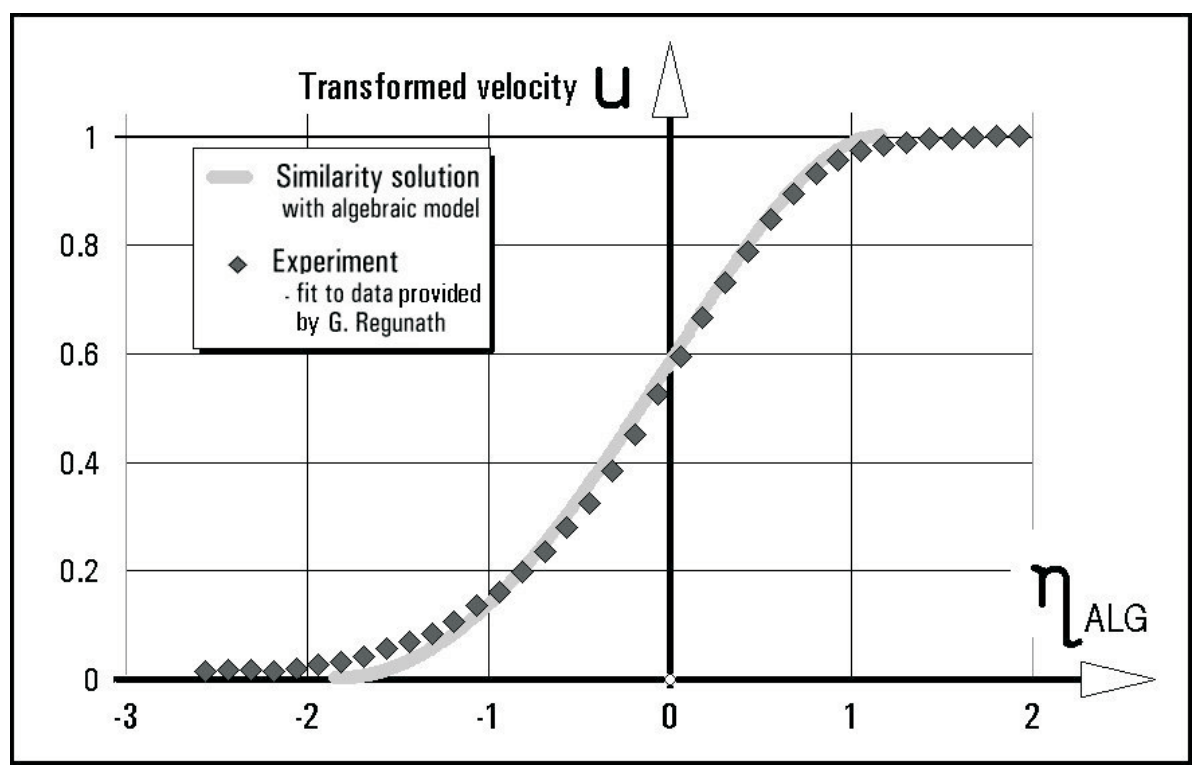

Fig. 18: Comparison of the profiles of relative velocity $u$ obtained by the present similarity solution using the algebraic model of turbulence with data points obtained as averages of the values at regular intervals in Fig. 13. 


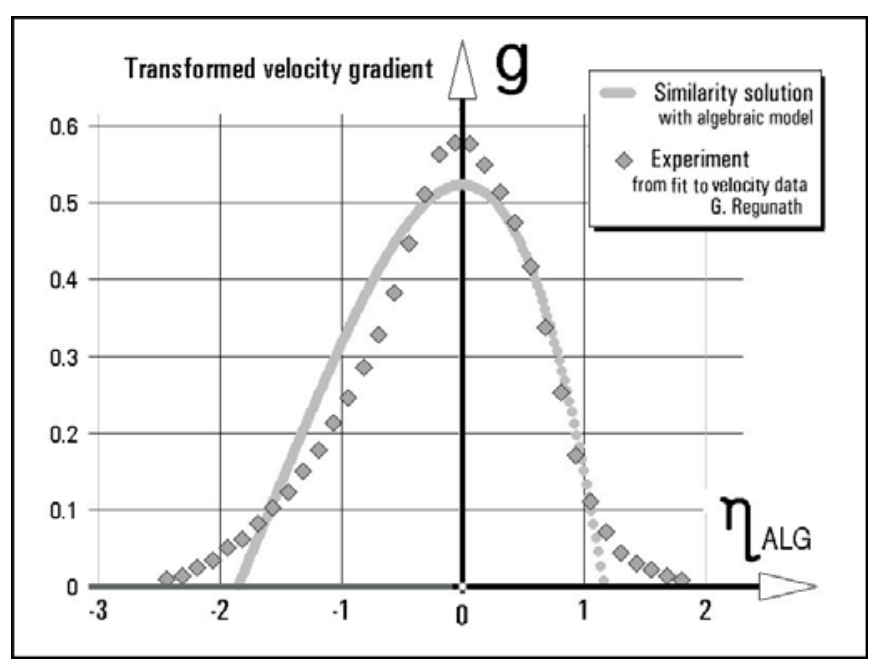

Fig. 19: Comparison of the profiles of the transversal gradient $\mathrm{d} u / \mathrm{d} \eta=g$ of the relative velocity $u$ obtained by the similarity solution and the experiment.

rium $\mathcal{P}=\in$ in the central part of the mixing layer. This means its local production rate is practically equal to the local destruction rate and does not generate any excess which has to be transported away. In the outer parts of the layer this ceases to be the case: the turbulence there is substantially influenced by transport (advective transport and/or gradient transport).

\subsection{1-Equation model}

This model of turbulence, based on simultaneous solution of an additional transport equation for one turbulence parameter, can perform what the algebraic model fails to do - to take into account the spatial transport of the turbulence. It should provide a better approximation to reality in the outer parts of the mixing layer, where the turbulence ceases to be in local equilibrium.

Again, it is useful to introduce (by analogy with [3, 5], and also with the already discussed algebraic model) the auxiliary variables. Apart from the dimensionless axial time-mean velocity $u$ and the dimensionless transversal gradient $g$, the similarity solution with the 1-equation model also operates with $\varepsilon$ - dimensionless specific energy of turbulent fluctuation, already defined by Eq.(25) and also uses its derivative $n=\frac{\mathrm{d} \varepsilon}{\mathrm{d} \eta}$.

The derivatives, of course, are now with respect to another independent variable, the transversal co-ordinate defined in Eq. (21). It should be noted that its definition operates with the constants $c_{z}, k$, and $s$.

Using the auxiliary variables, the equations derived for this model in part 2.3, Eqs. (29) and (30), are re-written as

$$
\begin{aligned}
& \frac{\mathrm{d} g}{\mathrm{~d} \eta}=-\frac{g f}{\sqrt{\varepsilon}}-\frac{g n}{2 \varepsilon} \\
& \frac{\mathrm{d} n}{\mathrm{~d} \eta}=\varepsilon \frac{c_{z}}{k s}-\frac{n f}{\sqrt{\varepsilon}}-\frac{n^{2}}{2 \varepsilon}-g^{2} \\
& \frac{\mathrm{d} f}{\mathrm{~d} \eta}=u \\
& \frac{\mathrm{d} u}{\mathrm{~d} \eta}=g \\
& \frac{\mathrm{d} \varepsilon}{\mathrm{d} \eta}=n
\end{aligned}
$$

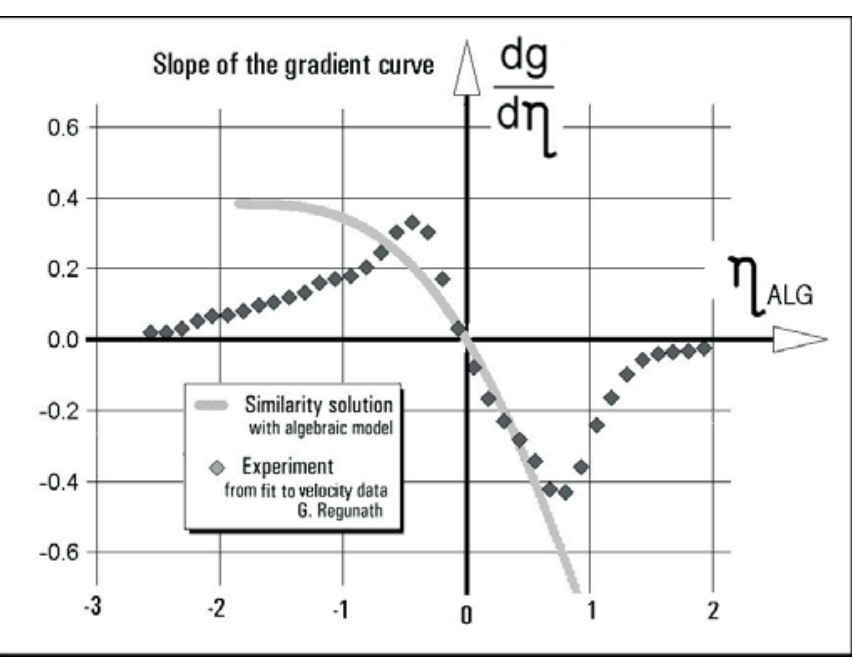

Fig. 20: Comparison of the profiles of $\mathrm{d} g / \mathrm{d} \eta=\mathrm{d}^{2} u / \mathrm{d} \eta^{2}$ obtained by the similarity solution and by the experiment. According to the primary alternative of Eq. (42), the values are also negative values of the transformed stream function $f$.

The problem now involves integration of these five simultaneous first-order ordinary differential equations, one of them containing a numerical parameter $c_{z} /(k s)$. The solution requires knowledge of five boundary conditions at a point, which is then used as the starting point of the integration. Also to be determined is the numerical value of the parameter $c_{z} /(k s)$. It may be useful to note that the same parameter was encountered in similarity solutions of a plane submerged jet in [3]. Its value evaluated there, from considering known jet spreading rates, was

$$
\frac{c_{z}}{k s}=6.40
$$

Unfortunately, the only points at which all five values $f, u$, $g, \varepsilon, n$ are known are the two extremes at the theoretical boundaries $\eta_{1 E Q} \rightarrow-\infty$ and $\eta_{1 E Q} \rightarrow+\infty$. These values (with zero gradients $g$, as well as $n$ ) are useless for starting the integration since they lead to the trivial case of both $\mathrm{d} g / \mathrm{d} \eta$ and $\mathrm{d} n / \mathrm{d} \eta$ remaining zero everywhere.

Investigation of similar problems with a hierarchy of models of progressively increasing complexity brings an important advantage, as already noted in similar circumstances in [2] and [5]: although the lower members of the hierarchy describe the reality less well, they operate with parameters easier to determine and to evaluate so that they provide useful starting points for more sophisticated approaches by the higher members of the hierarchy.

In the present case, the simple solution with the algebraic model - a model which may be, indeed, characterised as rather primitive, resulting in profiles with a discontinuity provides useful information which helps to solve the boundary conditions problem. It resulted above in the interesting conclusion that in the central part of the mixing layer the turbulence tends to be in local equilibrium, its local production rate being equal to the dissipation rate. The effect of the turbulence dissipation rate $\in$ in the transport equation for fluctuation energy - the second equation, for $\mathrm{d} n / \mathrm{d} \eta$, in Eq. (33) - is represented by the first term on the right-hand side. The last term on the right-hand side there represents the effect of turbulence production rate $P$ in the original trans- 
port Eq. (22). If the investigated turbulence were in equilibrium, $P=\in$, there would be

$$
g^{2}=\frac{\varepsilon c_{z}}{k s}
$$

production equals dissipation which may be differentiated to

$$
2 \frac{\mathrm{d} g}{\mathrm{~d} \eta} g=n \frac{c_{z}}{k s} \text {. }
$$

Inserting these two expressions into the equation for $\frac{\mathrm{d} g}{\mathrm{~d} \eta}-$ Eq. (43) - results in

$$
2 \frac{\mathrm{d} g}{\mathrm{~d} \eta}=-f \sqrt{\frac{c_{z}}{k s}}
$$

- which differs only by the constants (due to different definitions of the similarity transformed transverse variable $\eta$ ) from the equation $\mathrm{d} g / \mathrm{d} \eta=-f$ derived (Eq. (32)) for the algebraic model.

It is a reasonable choice - at least as an initial step to be improved upon later - to select again as the starting point of the integration, as in the algebraic model case, the central location at which there is $g \nabla=0$ and to use there as the boundary values for $u$ and $g$ the values which were successful with the algebraic model. The two remaining boundary conditions may then be, as the first approximation, evaluated for the equilibrium turbulence conditions:

$$
\begin{aligned}
& f=0 \\
& u=0.5834 \\
& g=0.523 \\
& \varepsilon=k s \frac{g^{2}}{c_{z}} \\
& n=2 \frac{\mathrm{d} g}{\mathrm{~d} \eta} g \frac{k s}{c_{z}}=0
\end{aligned}
$$

Also the constants of the problem can be reasonably identified using the previous, algebraic model solution.
Considering the definition of $\eta_{A L G}$ Eq. (15) and the value of the experimentally evaluated growth factor $s_{0.6}=0.1219$ in Fig. 14, the correspondence between the two similarity co-ordinates leads to the conclusion that the size $\ell$ of the vortices most effectively transporting momentum across the mixing layer is practically one half of the local convention thickness $\delta_{0.6}$ (cf. Eq. (7))

$$
k=0.50175 \text {. }
$$

This, together with $c_{z}=0.164$ from [2], indicates

$$
\frac{c_{z}}{k s}=2.6813
$$

- a value surprisingly smaller than 6.4 found for the jet flows, as mentioned above. A partial explanation for the difference is that the jet actually contains two shear layers, one on each side. Very rough reasoning would therefore suggest the parameter $c_{z} /(k s)$, reflecting the size of the turbulent eddies, to be $6.4 / 2=3.2$, smaller than but comparable to 2.6813 .

Finally, with the value of the Prandtl-Kolmogoroff coefficient $c_{v}=0.5477$ in definition Eq. (26) as derived e.g. in [2], it is possible to evaluate the coefficient in the present similarity transverse co-ordinate $\eta_{1 E Q}$ (which is needed e.g. for re-plotting the experimental data from Figs. 18 to 20 so that they may be used for comparison in Fig. 21).

$$
\eta_{1 E Q}=1.068593 \eta_{A L G} .
$$

Already at the very first attempt, numerical integration of Eqs. (33) using the conditions Eqs. (37) resulted in a surprisingly better approximation to the experimental data than the solution with the algebraic model, as shown for the quantities of the transformed momentum transport equation in Fig. 21. The transport effects now taken into account improve the character of the solution at and beyond the limits of the equilibrium zone, where the solution with the algebraic model resulted in the discontinuity and substantial divergence between the experiment and the theory - as shown e.g. in Fig. 20. Unfortunately, the integration run with the results shown in Fig. 21 did not meet the theoretical zero boundary

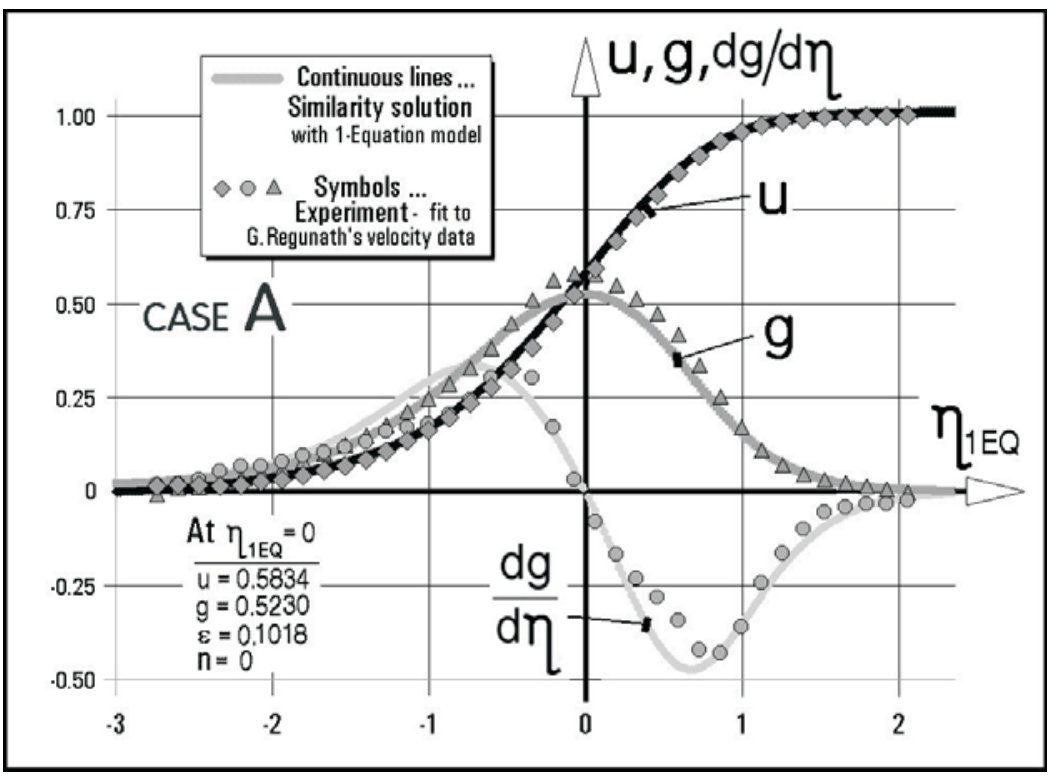

Fig. 21: Initial attempt at a solution using 1-equation model compared with the experiment. Profiles computed from the starting conditions at $\eta_{1 E Q}=0$ with the solution parameter $c_{z} /(k s)$ exactly as used earlier with the algebraic model for equilibrium turbulence - Eq. (47). 
conditions in what is assumed to be a non-turbulent external flow outside the layer for the fluctuation energy transport equation. Since the experimental data used for the comparison did not incorporate an information about the fluctuation energy, the success of the individual solution computations of the fluctuation transport equation was judged on the basis of whether or not they met the requirement of zero $\varepsilon$ values at the boundaries $\eta_{1 E Q} \rightarrow-\infty$ and $\eta_{1 E Q} \rightarrow+\infty$. Computation of the relative fluctuation energy profile $\varepsilon$ showed that in the case plotted in Fig. 21, which will be called case A, the boundary conditions were not met, and the fluctuation energy did not decrease to zero at the layer boundaries.

Of course, there is no reason why the conditions in the central part of the mixing layer, in the vicinity of the starting point of the integration, should be exactly in equilibrium. Using the equilibrium conditions Eq. (37) as the departure point for testing small deviations from them, a solution compliant with the theoretical zero turbulence condition at the boundaries was obtained. It was arrived at after rather laborious cut-and-try adjustments of the starting conditions as well as the parameter $c_{z} /(k s)$ of the solution. This theoretically proper solution is here described as case C. Fig. 22 presents for this case the computed profiles of the fluctuation energy as well as its transverse gradient in the similarity co-ordinates. The values of the starting conditions in what may be described as the "middle" of the mixing layer at $\eta_{1 E Q}=0$ are indicated both in Fig. 21 and in Fig. 22. While the changes of the other values are small and may be called insignificant, an important fact is that to obtain the theoretical boundary condition in case $\mathrm{C}$ a much smaller specific energy of fluctuations

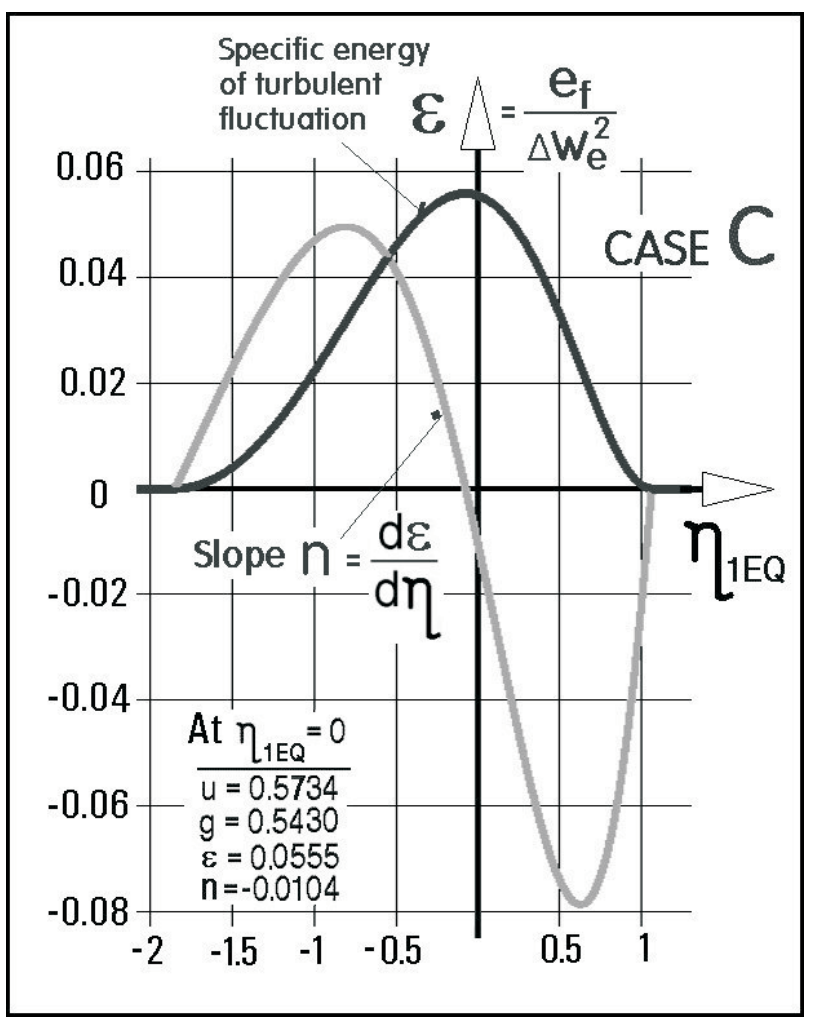

Fig. 22: Similarity transformed fluctuation energy profiles in the mixing layer obtained with the 1-equation model solution using the conditions at $\eta_{1 E Q}=0$ adjusted so that the integration proceeded to the ideal zero value boundary conditions on both sides of the layer was required, non-dimensionalised to e. It had to be decreased to nearly one half of the original value from Fig. 21 (comparison of Figs. 21 and 22 shows that the starting value $\varepsilon$ in case $\mathrm{C}$ had to be 0.545-times smaller in Fig. 21.

The general conclusion from the results in case $\mathrm{C}$ is that the distributions of the turbulence parameters - as well as e.g. of the transverse velocity gradient - exhibit much more abrupt ends at both boundaries than the experimental data which tend to approach the zero values asymptotically. Indeed, an important property of case $\mathrm{C}$ is the fact that instead of such an asymptotic character the distributions of the variables end at quite well defined boundary points - determined in Fig. 22 by the intersections of the slope curve with the horizontal axis. Further away, beyond these ends, the values cease to vary. The slope curves (not only that for the fluctuation energy in Fig. 22) exhibits a marked discontinuity in these end points.

This non-asymptotic character, with the rather abrupt ends of the solution, is not the only suspect feature of the 1-equation model solution with Tollmien's constant $\ell$ hypothesis applied to the characteristic scale of turbulence. A closer study of modelled turbulence reveals another strange aspect. Plotted in Fig. 23, there are distributions of individual terms in the transport equation for fluctuation energy $e_{f}$ across the mixing layer for the 1-equation solution with the starting values corresponding to case C. It is immediately apparent there that with the decreased starting value e the curve representing the turbulence dissipation term is very much lower than the curve of the production term. Contrary to what was deduced from the previous analysis of the experimental results and the 1-equation solution, the central part of the mixing layer is here not in local equilibrium $P=\in$. In fact, the imbalance between the production and dissipation is largest in the centre of the layer near $\eta_{1 E O}=0$. The excess of the produced and not dissipated turbulence is removed by the two transport mecha-

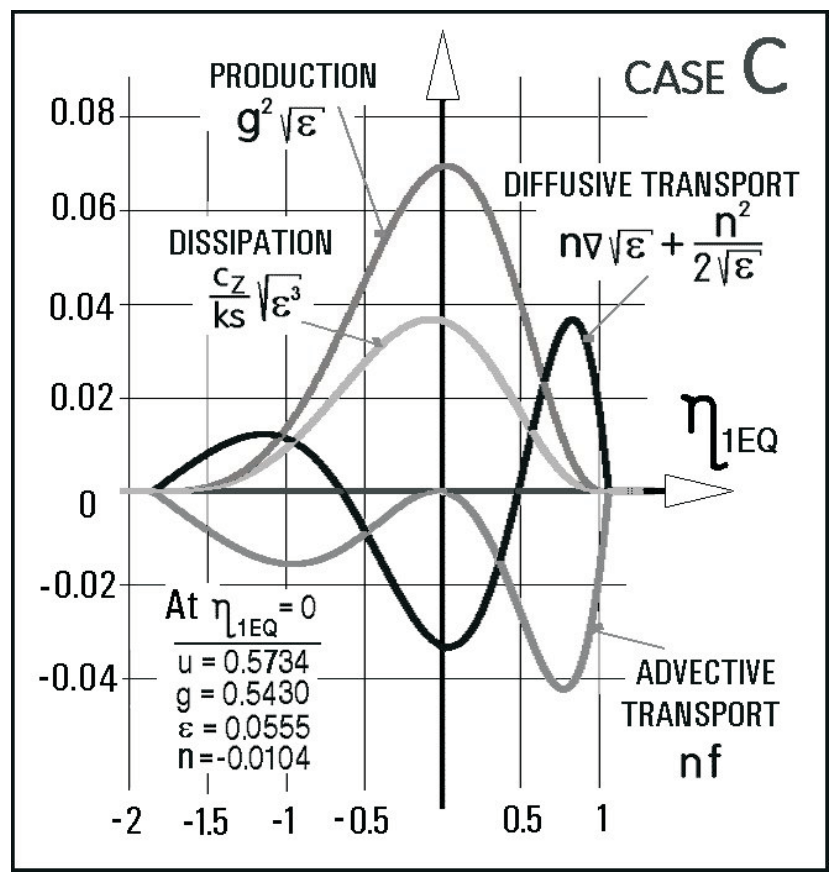

Fig. 23: Individual similarity transformed terms in the transport equation for fluctuation energy in the 1-equation model solution from Fig. 21 
nism, gradient diffusion and advection. The advective transport is necessarily zero near the centre of the layer, where there is no transversal fluid movement. Fig. 23 shows that indeed this is the location of extreme diffusion transport rate, the magnitude of which is exactly equal to the difference between the production and dissipation rates. In fact, there are two distinguishable components of the diffusive transport. The second, perhaps less obvious, component is due to the transversal variations of the turbulent viscosity. The present solution enables us to evaluate them independently - though to simplify the diagram Fig. 23 plots the sum of the two components as the overall diffusive transport (but note the indicated expression as the sum of the two terms).

As the advection increases with increasing distance $\eta_{1 E Q}$ from the centre, the absolute magnitude of the diffusion decreases. This trend does not end on reaching the zero value and continues so that the diffusion becomes positive at more distant locations from the central location. As the edges of the layer are approached, the diffusive transport is approximately equal in magnitude but of opposite sign to the advection. This mutual balancing is necessary since the difference between production and dissipation decreases, approaching zero at the layer edges. The strange fact is that in Fig. 23 there are actually no locations where the turbulence should be transported to. The solution in this case $\mathrm{C}$ does not predict any part of the layer cross section in which production is lower than dissipation. The quite massive diffusion and advection transports simply oppose each other.

This strange character is removed if we allow for the turbulence energy to be non-zero at least on one of the layer edges, as in case A described above. To find more about these

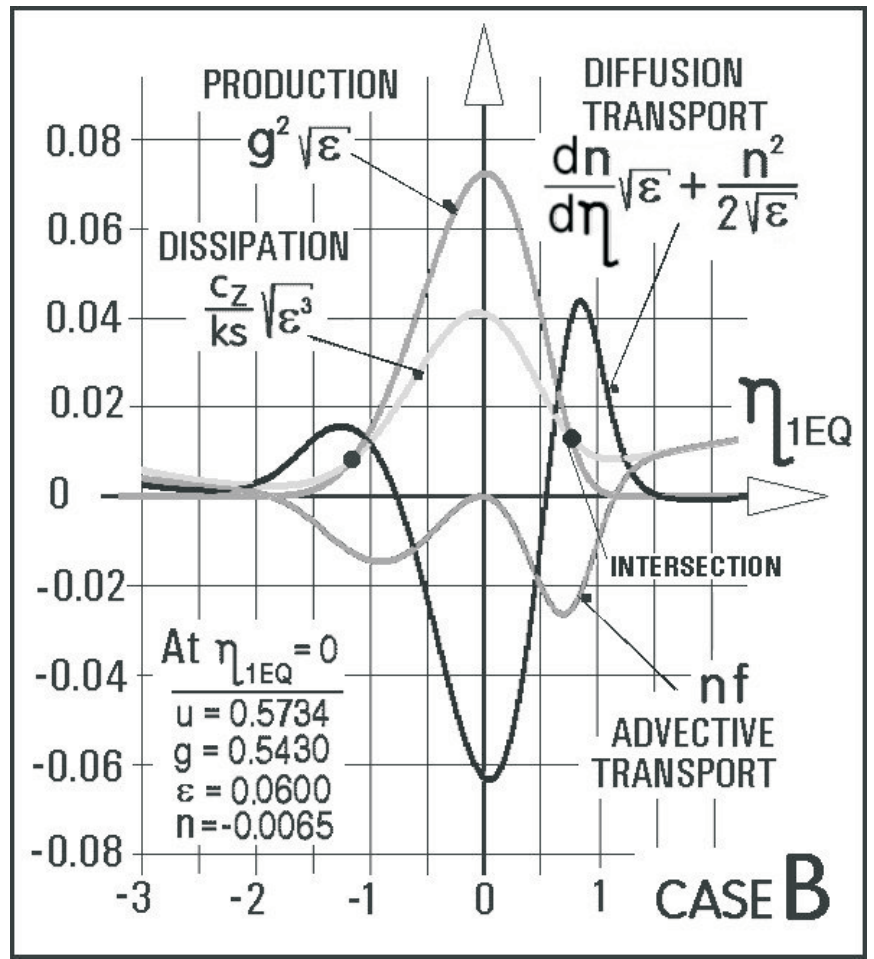

Fig. 24: Another 1-equation model solution, computed for a slightly increased starting value of fluctuation energy $\varepsilon$, between the values of case $\mathrm{A}$ and case $\mathrm{C}$. The diagram, by analogy with Fig. 23, again shows individual terms in the turbulent energy transport equation. aspects of the solution, yet another intermediate case B was evaluated. Essentially, it differed from case C by their higher starting value of transformed fluctuation energy $\varepsilon$, increased in case $\mathrm{B}$ to $\varepsilon=0.6$. The only other change in the starting conditions in case $\mathrm{B}$ was a decrease in the transformed gradient $n$. This change of the central slope was adjusted to obtain slightly higher turbulent transport towards the right-hand, higher velocity side. This corresponds to the idea of there being a higher turbulence inside the jet.

Plotted in Fig. 24, there are distributions of individual terms in the transport equation for the fluctuation energy $e_{f}$ across the mixing layer profiles for the 1-equation solution with the starting values corresponding to case B. In fact, the overall character of the curves does not differ very much from that seen in Fig. 23. Again, there is a difference - shown as the vertical distance - between the curve of the turbulence dissipation term and that of the production term. This difference, however, is now smaller. Evidently, the increase of the starting value of transformed fluctuation energy $\varepsilon$ shifts the conditions in the central zone of the mixing layer towards the local equilibrium $P=\in$. The important fact in Fig. 24 is the existence of the two intersection points - marked in the diagram by two black dots. The turbulence production is higher than the dissipation in the central zone up to these intersection points of the two curves. Further away it is dissipation that becomes dominant. This provides a sense to the idea of turbulence transport. Turbulent fluctuations are transported from the central zone, where they are produced, to the outer parts of the layer, where they are dissipated. It may be interesting to note that the transport into the outer parts - especially clearly seen on the right-hand side of the diagram in Fig. $24-$ is mainly the advective component of the transport. As a result of the presence of the turbulence in the outer parts, the abrupt ends at the layer edges seen in Fig. 23 now disappear, and the distributions of the flow parameters obtain an asymptotic character.

Unfortunately, as already mentioned, the experimental data collected by G. Regunath contain only the values of the time-mean velocities. There is no direct information about the turbulence, which would prove (or disprove) the present conclusions about the distributions of the turbulence equation terms. However, assuming validity of the 1-equation approach with the Tollmien hypothesis (the latter may be invalidated if it could be demonstrated that the turbulence length scale is not constant across the profiles), the nonzero outer turbulence level in the discussed cases A and B provides the only reasonable explanation for the experimental data.

This is demonstrated in Fig. 25, again (similarly as Fig. 20 for the algebraic model) providing a comparison of the second velocity derivative profiles $\frac{\mathrm{d} g}{\mathrm{~d} \eta}=\frac{\mathrm{d}^{2} u}{\mathrm{~d} \eta^{2}}=f(\eta)$ obtained by the experiment and by the similarity solution, here of course with the 1-equation model. Superimposed in the experimental data points (evaluated from G. Regunath's data by repeated numerical differentiation) are three curves, computed with three different sets of the starting values, corresponding to case A, case B and case C. It is immediately apparent that the theoretically "best" solution case C, meeting the condition of no turbulent fluctuation outside the mixing layer, fails to agree with the data points. In Fig. 25 it is seen to 


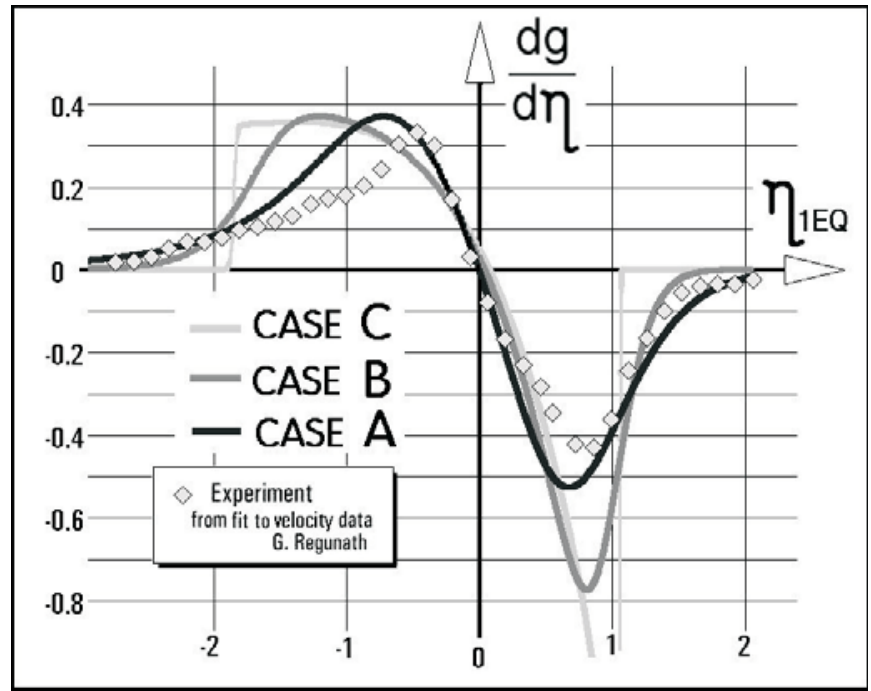

Fig. 25: Comparison of the profiles of $\frac{\mathrm{d} g}{\mathrm{~d} \eta}=\frac{\mathrm{d}^{2} u}{\mathrm{~d} \eta^{2}}$ evaluated (by differentiation) from the experimental velocity data and computed by the 1-equation model solution with the three cases differing in the numerical values of the conditions inserted at $\eta_{1 E Q}=0$ at the start of the integration.

almost coincide with the unrealistic end effects of the algebraic model solution in Fig. 20. The other computed cases in Fig. 25 form a succession of shapes progressing from the near-algebraic distribution to the distribution found in the experiment.

The inescapable evidence provided by these solutions is that the theoretically ideal 1-Equation solution incorporating the Tollmien hypothesis $\ell=$ const is inadequate when confronted with the available experimental data. Either it is to be admitted that turbulence is propagated away from the layer beyond its nominal boundaries or the size scale of the turbulence is distributed across the layer profiles in a more complex manner (which would be surprising, considering the almost perfect success of the hypothesis in the related solution of submerged turbulent jets, e.g. in [2, 5 and 6]).

Information about the distributions of the turbulence parameters across the mixing layer seems to be essential for deciding which is the correct road to follow. The author found some information in the experimental data of Liepmann \& Laufer [10] and Chow \& Korst [11], but they are insufficient and, indeed, in some aspects mutually contradictory.

An answer to the surprising findings about the inadequacy of what was considered the theoretically correct zero boundary conditions may be provided by the most complicated similarity solution based on solving two simultaneous transport equations for parameters characterising turbulence. This approach to the problem is the subject of another planned paper.

\section{Conclusions}

Several important results of the present investigations may be summarised as follows:

- The similarity transformation, converting the solution of two-dimensional flows into a problem described by a set of first-order ordinary differential equations, is a powerful tool deserving more attention.

- Its application to the present case of the mixing layer surrounding a round jet is successful. It proves the earlier conclusions from this author's 1978 experiments that the effects of transversal curvature may be neglected for engineering purposes.

- It is useful to use a hierarchy of progressively more complex turbulence models. Lower members of the hierarchy provide helpful departure points for solutions with more advanced models, providing insight and useful numerical values.

- It is useful to start the integration from the internal location where there is at least one zero gradient inside the layer.

- The algebraic as well as the 1-equation model solution described here compare favourably with the available experimental data and may suffice for practical applications. They are not perfect, in particular exhibiting physically unrealistic discontinuities at the edges and a number of open questions concerning the turbulence structure.

Solution with a more advanced, 2-equation turbulence model, is being prepared for a forthcoming publication.

\section{References}

[1] Tollmien, W.: "Berechnung turbulenter Ausbreitungsvorgänge." (Computation of Turbulent Propagation Processes - in German), Zeitschrift für Angewandte Mathematik und Mechanik, Vol. 6 (1926), p. 468.

[2] Tesař, V.: „Mezní vrstvy a turbulence“ (Shear Layers and Turbulence - in Czech), Publishing House of CTU Prague, Czech Republic, various editions from 1984 to 1996, ISBN 80-01-00675-1.

[3] Tesař, V.: "The Solution of the Plane Turbulent Jet." Acta Polytechnica, Vol. 36, (1996), No. 3, p. 15, ISSN 1210-2709.

[4] Tesař, V.: "Nozzle Characteristics - the Boundary Layer Model." Hydraulika i Pneumatika, Vol. XXIV (2004), No. 3, Poland, p. 35, ISSN 1505-3954.

[5] Tesař, V.: "Two-Equation Turbulence Model Solution of the Plane Turbulent Jet", Acta Polytechnica, Vol. 35 (1995), No. 2, p. 19, ISSN 1210-2709.

[6] Tesař, V.: "Two-Equation Turbulence Model Similarity Solution of the Axisymmetric Fluid Jet." Acta Polytechnica Vol. 41 (2001), No. 2, p. 26, ISSN 1210-2709.

[7] Regunath, G., Tesař, V., Zimmerman, W. J. B., Russell, N.: "Novel Merging Swirl Burner Design Controlled by Helical Mixing." Proceedings of the $7^{\text {th }}$ World Congress of Chemical Engineering, Glasgow, 2005.

[8] Görtler, H.: "Berechnung von Aufgaben der freien Turbulenz auf Grund eines neuen Näherungsansatzes." (Computation of Free Turbulence Problems on the Basis of a New Approximation Theorem - in German) Zeitschrift für Angewandte Mathematik und Mechanik, Vol. 22 (1942), p. 244.

[9] Tesař, V.: "Similarity Solutions of Basic Turbulent Shear Flows with One- and Two-Equation Models of Turbulence." Zeitschrift für Angewandte Mathematik und Mechanik, Vol. 77 (1997), Sup. 1, p. 333, ISSN 0946-8463. 
[10] Liepmann, H. W., Laufer, J.: "Investigation of Free Turbulent Mixing”, NACA Technical Note TN 1257, 1947.

[11] Chow, W. L., Korst, H. H.: "On the Flow Structure within a Constant Pressure Compressible Turbulent Jet Mixing Region." NASA Technical Note D-1894, Ames Research Center, 1963.

[12] Cheng, B. L., Glimm, J., Jin, H. S., Sharp D.: "Theoretical Methods for the Determination of Mixing." Laser and Particle Beams, Vol. 21 (2003), Issue 03, p. 429.

[13] Olsen, M. G., Dutton, J. C.: "Planar Velocity Measurements in a Weakly Compressible Mixing Layer." Journal of Fluid Mechanics, Vol. 486 (2003), p. 51.

[14] Tesař, V.: „Směšování koaxiálních průtoků a fluidická čerpadla." (Mixing of Concurrent Flows and Fluidic Pumps - in Czech), Acta Polytechnica (1982), No. 7, II, 1.

[15] Tesař, V.: "Time-Mean Helicity Distribution in Turbulent Swirling Jets." accepted for publication in Acta Polytechnica, 2005, ISSN 1210-2709

[16] Noether, E.: "Invariante Variationsprobleme." Nachr. König. Gessell. Wissen. Göttingen, Math-Phys. Kl., 1918, p. 235.
[17] Kara, A. H., Mahomed, F. M.: "Relation between Symmetries and Conservation Laws." Internat. Journ. Theoret. Phys., Vol. 39 (2000), p. 23.

[18] Ibragimov, N. Kh.: Elementary Lie Group Analysis and Ordinary Differential Equations. Chichester, New York: Wiley 1999, ISBN 0471974307.

[19] Tesař, V.: "Valvole fluidiche senza parti mobili." (No-Moving-Part Fluidic Valves - in Italian), Oleodinamica - pneumatica, Vol. 39 (1998), No. 3, p. 216, ISSN 1 122-5017.

Prof. Ing. Václav Tesař, CSc.

e-mail:v.tesar@sheffield.ac.uk

University of Sheffield

Mappin Street S1 3JD

Sheffield, UK

AV ČR

Dolejškova 5

18200 Prague 8, Czech Republic 